\title{
Decision making with imprecise probabilities and utilities by means of statistical preference and stochastic dominance
}

\author{
Ignacio Montes ${ }^{1}$, Enrique Miranda ${ }^{1}$, Susana Montes ${ }^{1}$ \\ ${ }^{a}$ University of Oviedo, Dep. of Statistics and O.R.
}

\begin{abstract}
A problem of decision making under uncertainty in which the choice must be made between two sets of alternatives instead of two single ones is considered. A number of choice rules are proposed and their main properties are investigated, focusing particularly on the generalizations of stochastic dominance and statistical preference. The particular cases where imprecision is present in the utilities or in the beliefs associated to two alternatives are considered.
\end{abstract}

Keywords: Preference learning, binary relations, stochastic dominance, statistical preference, random sets, imprecise probabilities.

\section{Introduction}

In decision making under uncertainty, it is not uncommon to encounter situations with vague or conflicting information about the probabilities or the utilities associated to the different alternatives. We may think for instance of conflicts among the opinions of several experts, limits or errors in the observational process, or simply partial or total ignorance about the process underlying the alternatives. In any such case, the elicitation of an unique probability/utility model for each of the alternatives may be difficult and its use, questionable.

One of the solutions that have been proposed for situations like this is to consider a robust approach, by means of a set of probabilities and utilities. The use of this approach to compare two alternatives is formally equivalent to the comparison of two sets of alternatives, those associated to each possible probability-utility pair. Hence, it becomes useful to consider comparison methods that allow us to deal with sets of alternatives instead of single ones. However, the way to compare of sets of alternatives is no longer immediate: we may compare all possibilities within each of the sets, or also select some particular elements of each set, to take into account phenomena of risk aversion, for instance. This gives rise to a number of possibilities. Moreover, even in the

*Corresponding author. Tel: (+34) 985186466, Fax: $(+34) 985103353$ 
simpler case where we choose one alternative from each set, we must still decide which criterion we shall consider to determine the preferred one.

There is quite an extensive literature on how to deal with imprecise beliefs and utilities when our choice is made by means of an expected utility model [? ? ? ? ]. However, the problem has almost remained unexplored for other choice functions. In this paper we focus mostly on two different optimality criteria that serve as an alternative to the expected utility model: stochastic dominance and statistical preference. The former is based on the comparison of the distribution functions associated to the alternatives, and has been applied in economics [? ? ]; the latter can be seen as a robust alternative to expected utility which is based on the median instead of the mean as a location parameter, and was introduced in [? ], as an equivalent and graded version of the method presented in [? ]; it is also a counterpart of the expected utility model when the rewards of the different alternatives are expressed in a qualitative scale [? ]. We shall recall the basic aspects of these two criteria in Section ??.

In Section ??, we define a number of choice models for sets of alternatives starting from some binary relation, based on earlier work on this problem carried out in [? ], and apply them to the particular cases where this relation is the one associated to stochastic dominance or statistical preference. Then we consider two particular cases: first, in Section ?? we deal with the case where we have precise information about the beliefs but imprecise one about the utilities. We model this situation by means of multi-valued mappings, or random sets [? ] which, under the epistemic interpretation considered in [? ], can be seen as collections of random variables imprecisely specified. We show that under some conditions the comparison can be simplified using the lower and upper probabilities induced by the random set. Secondly, we consider in Section ?? the case where we have precise utilities but imprecise beliefs, and show that there are two additional notions that may be more useful in such a scenario.

The different conditions and their properties are illustrated by means of an example in Section ??. We conclude the paper by giving a number of additional remarks in Section ??.

\section{Preliminary concepts}

Let us review the basics about the two optimality criteria for decision making under uncertainty we shall consider in this paper. To clarify our set up, we consider a problem where we must choose between alternatives $X, Y$ whose utilities depend on the values $\omega$ of the states of nature. We assume that we have probabilistic information about these states of nature, so that $X, Y$ are defined as variables from a probability space $(\Omega, \mathcal{A}, P)$ and taking values on an utility space $\Omega^{\prime}$. For the most part, we shall assume that $\Omega^{\prime}$ is a bounded

subset of the reals; however, in the case of statistical preference we may have qualitative utilities, and then $\Omega^{\prime}$ may correspond to an ordered qualitative scale. 


\subsection{Stochastic dominance}

The notion of stochastic dominance between random variables is based on the comparison of their corresponding distribution functions. Assume that our utility scale is $\Omega^{\prime}=[0,1]$ (the results in this section generalize immediately to the case where $\Omega^{\prime}$ is any bounded interval of real numbers). Distribution functions are thus defined in the following way:

Definition 1. A cumulative distribution function on $[0,1]$ is a function $F$ : $[0,1] \rightarrow[0,1]$ satisfying the following properties:

- $x \leq y \Rightarrow F(x) \leq F(y) \forall x, y$ [Monotonicity].

- $F(1)=1$ [Normalization].

- $F(x)=\lim _{\epsilon \downarrow 0} F(x+\epsilon) \forall x<1$ [Right-continuity].

Any $F$ satisfying the properties of monotonicity and normalization is associated to a finite additive probability measure, and we shall call it a finitely additive distribution function.

One of the most popular methods for the comparison of cumulative distribution functions is stochastic dominance [? ]:

Definition 2. Given two cumulative distribution functions $F$ and $G$, we say that $F$ stochastically dominates $G$, and denote it $F \succeq_{\mathrm{FSD}} G$, if $F(t) \leq G(t)$ for every $t$ in [0,1], and given two random variables $X, Y$ taking values on $[0,1]$, we say that $X$ stochastically dominates $Y$, and denote it $X \succeq_{\mathrm{FSD}} Y$ when its associated distribution function $F_{\mathrm{X}}$ stochastically dominates $F_{\mathrm{Y}}$, where

$$
F_{\mathrm{X}}(t)=P(X \leq t) \text { and } F_{\mathrm{Y}}(t)=P(Y \leq t) \forall t \in[0,1] .
$$

In the literature, this notion is sometimes called first degree stochastic dominance, in order to distinguish it from a number of weaker conditions called second, third,... degree stochastic dominance [? ]. This is the reason of the notation $\succeq_{\text {FSD }}$. Occasionally the notation $\succeq_{s t}$ is also employed (see for instance [?]).

This definition induces a partial order in the space $\mathbb{F}$ of cumulative distribution functions, from which we can derive the notions of strict stochastic dominance, indifference and incomparability:

- We say that $F$ stochastically dominates $G$ strictly, and denote it by $F \succ_{\text {FSD }} G$, if $F \succeq_{\text {FSD }} G$ but $G \succeq_{\text {FSD }} F$. This holds if and only if $F \leq G$ and there is some $t \in[0,1]$ such that $F(t)<G(t)$.

- $F$ and $G$ are stochastically indifferent, and denote it by $F \equiv_{\mathrm{FSD}} G$, if $F \succeq_{\text {FSD }} G$ and $G \succeq_{\text {FSD }} F$, or equivalently, if $F=G$.

- $F$ and $G$ are stochastically incomparable, and denote it by $F \nsim_{\mathrm{FSD}} G$, if $F \nsucceq_{\mathrm{FSD}} G$ and $G \succeq_{\mathrm{FSD}} F$. 
Thus, (F, $\left.\succ_{\mathrm{FSD}}, \equiv_{\mathrm{FSD}}, \nsim_{\mathrm{FSD}}\right)$ constitutes a preference structure ([?]).

Stochastic dominance is commonly used in economics and finance [? ? ] and can be given the following interpretation: $F \succeq_{\text {FSD }} G$ means that the choice of $F$ over $G$ is rational, in the sense that we prefer the alternative with greater probability of providing a utility above a certain threshold $t$, and this for all possible $t$. The notion has also been used in other frameworks such as reliability theory, statistical physics, epidemiology, etc. We refer to [? ? ] for more information, and to [? ? ] for recent works in the context of decision making. It is characterized by the following property:

Theorem 1. [? ] Given two random variables $X$ and $Y$ it holds that: $X \succeq_{\text {FSD }}$ $Y$ if and only if $E(u(X)) \geq E(u(Y))$ for every non-decreasing $u$.

The result is based on the equivalence between $X \succeq_{\mathrm{FSD}} Y$ and the inequality $E\left(I_{[t, \infty)}(X)\right) \geq E\left(I_{[t, \infty)}(Y)\right) \forall t \in \mathbb{R}$, so if we denote by $\mathcal{U}^{*}$ the set of nondecreasing and bounded maps from $\mathbb{R}$ to $\mathbb{R}$, we may also characterize stochastic dominance by:

$$
X \succeq_{\mathrm{FSD}} Y \Leftrightarrow E(u(X)) \geq E(u(Y)) \text { for every } u \in \mathcal{U}^{*} .
$$

\subsection{Statistical preference}

We next introduce the notion of statistical preference. One of its advantages is that it is applicable to variables $X, Y$ taking values on any ordered qualitative scale $\Omega^{\prime}$, which need not be numerical. It is based on the notion of probabilistic relation.

Definition 3. [? ] Given a set of alternatives $\mathcal{D}$, a probabilistic relation is a map $Q: \mathcal{D} \times \mathcal{D} \rightarrow[0,1]$ satisfying $Q(a, b)+Q(b, a)=1$ for all $a, b$ in $\mathcal{D}$.

Consider two variables $X, Y$ from a probability space $(\Omega, \mathcal{A}, P)$ to an ordered utility space $\Omega^{\prime}$ and define

$$
Q(X, Y)=P(X>Y)+\frac{1}{2} P(X=Y) ;
$$

then it is easy to see that $Q$ is a probabilistic relation. The value $Q(X, Y)$ can be interpreted as a measure of the strength of our preference of $X$ over $Y$. Statistical preference can then be introduced as a decision criterion based on this probabilistic relation:

Definition 4. [? ? ] We say that the random variable $X$ is:

- statistically preferred to $Y$, and denote it by $X \succeq_{\mathrm{SP}} Y$, if $Q(X, Y) \geq \frac{1}{2}$;

- strictly statistically preferred to $Y\left(X \succ_{\mathrm{SP}} Y\right)$ if $Q(X, Y)>\frac{1}{2}$;

- statistically indifferent to $Y\left(X \equiv_{\mathrm{SP}} Y\right)$ when $Q(X, Y)=\frac{1}{2}$. 
Note that, if $\mathcal{D}$ denote a set of random variables defined on the probability space, $\left(\mathcal{D}, \succ_{\mathrm{SP}}, \equiv_{\mathrm{SP}}\right)$ constitutes a preference structure without incomparable elements.

Remark 1. One context where statistical preference appears naturally is that of decision making with qualitative random variables. Dubois et al. show in [?] that given two variables $X, Y: \Omega \rightarrow \Omega^{\prime}$, where $\left(\Omega^{\prime}, \succeq_{\Omega^{\prime}}\right)$ is an ordered qualitative scale, then, given a number of rationality axioms over our decision rule, the choice between $X$ and $Y$ must be made by means of the likely dominance rule, which says that $X$ is preferred to $Y$ if and only if $\left[X \succeq_{\Omega^{\prime}} Y\right] \succsim\left[Y \succeq_{\Omega^{\prime}} X\right]$, where

$$
\begin{aligned}
& {\left[X \succeq_{\Omega^{\prime}} Y\right]=\left\{\omega \in \Omega: X(\omega) \succeq_{\Omega^{\prime}} Y(\omega)\right\} \text { and }} \\
& {\left[Y \succeq_{\Omega^{\prime}} X\right]=\left\{\omega \in \Omega: Y(\omega) \succeq_{\Omega^{\prime}} X(\omega)\right\}}
\end{aligned}
$$

and where $\succsim$ is a binary relation on subsets of $\Omega$. One of the most interesting cases is that where $\succsim$ is determined by a probability measure $P$, so $A \succsim B \Leftrightarrow$ $P(A) \geq P(B)$; we obtain that $X$ is preferred to $Y$ if and only if $P(X \geq Y) \geq$ $P(Y \geq X)$, or, equivalently, if $X \succeq_{\mathrm{SP}} Y$. Hence, we can see statistical preference as a natural generalization of the expected utility model to the case of qualitative utilities.

Statistical preference is also applicable when we are dealing with quantitative utilities and the utility scale $\Omega^{\prime}$ is a subset of the reals; in that case, statistical preference is related to a location parameter: the median. This is detailed in [? , Theorem 5].

More generally, the idea of statistical preference is to consider $X$ preferred to $Y$ when it provides greater utility the majority of times. As such, it is close to the rule of majority in voting systems; taking into account Condorcet's paradox (see [?]) it is not surprising then that the binary relation associated to statistical preference is not transitive: it is possible to find random variables $X, Y$ and $Z$ satisfying $X>_{\mathrm{SP}} Y, Y>_{\mathrm{SP}} Z$ and $Z>_{\mathrm{SP}} X$. Let us recall one example, that shall be used later on; another one can be found in [? , Example 3].

Example 1. [? ] Consider independent dice (here, we call dice a random variable with a discrete uniform distribution over a six-value space) defined by:

$$
\begin{aligned}
& X=\{1,3,4,15,16,17\} . \\
& Y=\{2,10,11,12,13,14\} . \\
& Z=\{5,6,7,8,9,18\} .
\end{aligned}
$$

It holds that $Q(X, Y)=\frac{20}{36}, Q(Y, Z)=\frac{25}{36}$, but $Q(Z, X)=\frac{21}{36}$.

The binary relation induced by statistical preference is reflexive, because we see immediately from Eq. (??) that $Q(X, X)=\frac{1}{2}$, and therefore $X \equiv_{\mathrm{SP}} X$ for every $X$. However, it is not antisymmetric, since $X \equiv_{\mathrm{SP}} Y \Leftrightarrow Q(X, Y)=\frac{1}{2}$, and this does not imply that $X=Y$ : to see an example, consider $\Omega=\left\{\omega_{1}, \omega_{2}\right\}$, with $P\left(\left\{\omega_{i}\right\}\right)=\frac{1}{2}$, and the variables $X, Y$ given by

\begin{tabular}{c|cc} 
& $\omega_{1}$ & $\omega_{2}$ \\
\hline$X$ & 2 & 0 \\
$Y$ & 1 & 1
\end{tabular}


Then, $Q(X, Y)=\frac{1}{2}$, and consequently $X \equiv_{\mathrm{SP}} Y$ even if the two random variables are different.

With respect to the expected utility, statistical preference can be characterized in the following way [? ]. Given two real-valued random variables $X, Y$,

$$
X \succeq_{\mathrm{SP}} Y \Leftrightarrow X-Y \succeq_{\mathrm{SP}} 0 \Leftrightarrow E(u(X-Y)) \geq 0,
$$

where the utility function $u: \mathbb{R} \rightarrow \mathbb{R}$ is given by $u=I_{(0,+\infty)}-I_{(-\infty, 0)}$.

\subsection{Imprecise probabilities}

To conclude this part, we discuss briefly imprecise probability models. This is the generic term used to refer to all mathematical models that serve as an alternative and a generalization to probability models in cases of imprecise knowledge. It includes possibility measures [? ], Choquet capacities [? ], belief functions [? ] or coherent lower previsions [? ], among others.

In this paper, we shall use imprecise probability models because we shall deal with a set $\mathcal{X}$ of alternatives, each with its corresponding probability distribution; we obtain thus a set $\mathcal{P}$ of probability measures. This set can be summarized by means of its lower and upper envelopes, which are given by:

$$
\underline{P}(A):=\inf _{P \in \mathcal{P}} P(A), \bar{P}(A):=\sup _{P \in \mathcal{P}} P(A),
$$

and which are coherent lower and upper probabilities in the sense of Walley [? ]. If instead we consider the lower and upper expectation operators, given by $\underline{E}_{\mathcal{P}}(f):=\inf _{P \in \mathcal{P}} E_{P}(f), \bar{E}_{\mathcal{P}}(f):=\sup _{P \in \mathcal{P}} E_{P}(f)$, we obtain coherent lower and upper previsions. Conversely, if we specify a coherent lower probability $\underline{P}$, we shall denote by $\mathcal{M}(\underline{P}):=\{P$ probability measure $: P(A) \geq \underline{P}(A) \forall A\}$ its associated credal set.

In a similar way, if we consider a set $\mathcal{F}$ of distribution functions, its associated lower and upper distribution functions are given by

$$
\underline{F}(x):=\inf _{F \in \mathcal{F}} F(x), \quad \bar{F}(x):=\sup _{F \in \mathcal{F}} F(x) .
$$

Then $\underline{F}, \bar{F}$ are finitely additive distribution functions, and the set of distribution functions bounded between them is called a $p$-box [?], and denoted $(\underline{F}, \bar{F})$. Pboxes shall be useful when dealing with imprecise stochastic dominance.

\section{Comparison of pairs of sets of random variables}

In the following, we propose a number of comparison methods for pairs of sets of variables which are based on performing pairwise comparisons of elements within these sets. We shall first give our definitions for the case where the comparisons of the elements are made by means of a binary relation, and later apply them to the particular cases where this binary relation consists of stochastic dominance or statistical preference. Some of the ideas within this section are similar to those put forward in [? ] when extending stochastic dominance in order to deal with imprecise information. 
Definition 5. Let $\succeq$ be a binary relation on the set of variables from a probability space $(\Omega, \mathcal{A}, P)$ to an ordered utility scale $\Omega^{\prime}$. Given two sets of random variables $\mathcal{X}$ and $\mathcal{Y}$, we say that:

1. $\mathcal{X} \succeq_{1} \mathcal{Y}$ if and only if for every $X \in \mathcal{X}, Y \in \mathcal{Y}$ it holds that $X \succeq Y$.

2. $\mathcal{X} \succeq_{2} \mathcal{Y}$ if and only if there is some $X \in \mathcal{X}$ such that $X \succeq Y$ for every $Y \in \mathcal{Y}$.

3. $\mathcal{X} \succeq_{3} \mathcal{Y}$ if and only if for every $Y \in \mathcal{Y}$ there is some $X \in \mathcal{X}$ such that $X \succeq Y$.

4. $\mathcal{X} \succeq_{4} \mathcal{Y}$ if and only if there are $X \in \mathcal{X}, Y \in \mathcal{Y}$ such that $X \succeq Y$.

5. $\mathcal{X} \succeq_{5} \mathcal{Y}$ if and only if there is some $Y \in \mathcal{Y}$ such that $X \succeq Y$ for every $X \in \mathcal{X}$

6. $\mathcal{X} \succeq_{6} \mathcal{Y}$ if and only if for every $X \in \mathcal{X}$ there is $Y \in \mathcal{Y}$ such that $X \succeq Y$.

The relationships between the definitions are summarized in the following proposition. Its proof is immediate and therefore omitted.

Proposition 2. The following implications hold:

(a) $\succeq_{1} \Rightarrow_{2} \Rightarrow \succeq_{3} \Rightarrow \succeq_{4}$.

(b) $\succeq_{1} \Rightarrow_{5} \Rightarrow \succeq_{6} \Rightarrow \succeq_{4}$.

The previous implications can also be seen easily in the particular case where $\mathcal{X}$ and $\mathcal{Y}$ are finite sets, $\mathcal{X}=\left\{X_{1}, \ldots, X_{n}\right\}$ and $\mathcal{Y}=\left\{Y_{1}, \ldots, Y_{m}\right\}$. Denote by $M$ the $n \times m$ matrix where $M_{i, j}=1$ if $X_{i} \succeq Y_{j}$ and 0 otherwise. The above definitions are characterized in the following way:

- $\mathcal{X} \succeq_{1} \mathcal{Y} \Leftrightarrow M=1$.

- $\mathcal{X} \succeq_{2} \mathcal{Y} \Leftrightarrow \exists i \in\{1, \ldots, n\}$ s.t. $M_{i, .}=1$.

- $\mathcal{X} \succeq_{3} \mathcal{Y} \Leftrightarrow \nexists j \in\{1, \ldots, m\}$ s.t. $M_{\cdot, j}=0$.

- $\mathcal{X} \succeq_{4} \mathcal{Y} \Leftrightarrow M \neq 0$.

- $\mathcal{X} \succeq_{5} \mathcal{Y} \Leftrightarrow \exists j \in\{1, \ldots, m\}$ s.t. $M_{\cdot, j}=1$.

- $\mathcal{X} \succeq_{6} \mathcal{Y} \Leftrightarrow \nexists i \in\{1, \ldots, n\}$ s.t. $M_{i, \cdot}=0$.

Remark 2. The ideas in the above definition are somewhat similar to those in the field of robust ordinal regression [? ? ? ]: in that case we consider a set of additive value functions compatible with our partial preferences, and the problem of considering a choice function that is robust with respect to this information is considered. In particular, in [? ] the best and the worst expected rewards for any alternative with respect to the set of value functions are considered. This could be embedded into our formulation above, provided that: (a) our binary relation is that associated to expected utility; (b) the set of alternatives corresponds to the conjunction of an alternative with a set of value functions. See also Section ?? later on. 
Observe that for any binary relation $\succeq$, its extensions $\succeq_{2}$ and $\succeq_{3}$ are quite similar: both compare the best alternatives within each set $\mathcal{X}, \mathcal{Y}$. The difference between them lies on whether there is a maximal element within each of these sets or not. A similar comment (this time in terms of the worst alternatives) can be made for $\succeq_{5}, \succeq_{6}$. Taking this into account, we can easily give a necessary and sufficient condition for the equivalences $\succeq_{2} \Leftrightarrow \succeq_{3}$ and $\succeq_{5} \Leftrightarrow \succeq_{6}$.

Proposition 3. Let $\succeq$ be a binary relation on the set of random variables that is reflexive and transitive.

(a) Given a set $\mathcal{X}$ of random variables, $\mathcal{X} \succeq_{3} \mathcal{Y} \Rightarrow \mathcal{X} \succeq_{2} \mathcal{Y}$ for any set of variables $\mathcal{Y}$ if and only if $\mathcal{X}$ has a maximum element under $\succeq$.

(b) Given a set $\mathcal{Y}$ of random variables, $\mathcal{X} \succeq_{6} \mathcal{Y} \Rightarrow \mathcal{X} \succeq_{5} \mathcal{Y}$ for any set of variables $\mathcal{X}$ if and only if $\mathcal{Y}$ has a minimum element under $\succeq$.

Proof. (a) Assume that $\mathcal{X}$ has a maximum element $X$ such that $X \succeq X^{\prime}$ for every $X^{\prime} \in \mathcal{X}$. If $\mathcal{X} \succeq_{3} \mathcal{Y}$, then for every $Y \in \mathcal{Y}$ there is some $X_{Y} \in \mathcal{X}$ such that $X_{Y} \succeq Y$. Since $\succeq$ is transitive and $X \succeq X_{Y}$, we deduce that $X \succeq Y$ for every $Y \in \mathcal{Y}$, and as a consequence $\mathcal{X} \succeq_{2} \mathcal{Y}$.

Conversely, if $\mathcal{X}$ does not have a maximum element, we can take $\mathcal{Y}=\mathcal{X}$ and we would have $\mathcal{X} \equiv_{3} \mathcal{Y}$ because $\succeq$ is reflexive; however, $\mathcal{X}$ and $\mathcal{Y}$ are incomparable with respect to $\succeq_{2}$ because $\mathcal{X}$ does not have a maximum element.

(b) Similarly, if $\mathcal{Y}$ has a minimum element $Y$, it holds that $Y^{\prime} \succeq Y$ for any $Y^{\prime} \in \mathcal{Y}$. If $\mathcal{X} \succeq_{6} \mathcal{Y}$, then for every $X \in \mathcal{X}$ there exists $Y_{X} \in \mathcal{Y}$ such that $X \succeq Y_{X}$, and since $\succeq$ is transitive and $Y_{X} \succeq Y$ we obtain that $X \succeq Y$ for every $X \in \mathcal{X}$, whence $\mathcal{X} \succeq_{5} \mathcal{Y}$.

Conversely, if $\mathcal{Y}$ does not have a minimum element, we can take $\mathcal{X}=\mathcal{Y}$ and we would have $\mathcal{X} \equiv_{6} \mathcal{Y}$ because $\succeq$ is reflexive; however, $\mathcal{X}$ and $\mathcal{Y}$ are incomparable with respect to $\succeq_{5}$ because $\mathcal{Y}$ does not have a minimum element.

Under some conditions, we can also give a simpler characterization of the above conditions:

Proposition 4. Let $\succeq$ be a binary relation between random variables, and assume that it satisfies the Pareto Dominance condition:

$$
X(\omega) \geq Y(\omega) \forall \omega \Rightarrow X \succeq Y
$$

Consider two sets of random variables $\mathcal{X}, \mathcal{Y}$. If the random variables min $\mathcal{X}$, $\max \mathcal{X}$ belong to $\mathcal{X}$ and $\min \mathcal{Y}, \max \mathcal{Y}$ belong to $\mathcal{Y}:$

(a) $\mathcal{X} \succeq_{1} \mathcal{Y} \Leftrightarrow \min \mathcal{X} \succeq \max \mathcal{Y}$.

(b) $\mathcal{X} \succeq_{2} \mathcal{Y} \Leftrightarrow \mathcal{X} \succeq_{3} \mathcal{Y} \Leftrightarrow \max \mathcal{X} \succeq \max \mathcal{Y}$.

(c) $\mathcal{X} \succeq_{4} \mathcal{Y} \Leftrightarrow \max \mathcal{X} \succeq \min \mathcal{Y}$.

(d) $\mathcal{X} \succeq_{5} \mathcal{Y} \Leftrightarrow \mathcal{X} \succeq_{6} \mathcal{Y} \Leftrightarrow \min \mathcal{X} \succeq \min \mathcal{Y}$. 
Proof. The result follows from Definition ?? and Proposition ??, taking into account that when both $\mathcal{X}, \mathcal{Y}$ include a maximum and a minimum random variable, Eq. (??) implies that for every $X \in \mathcal{X}, Y \in \mathcal{Y}$,

$$
\begin{gathered}
\min \mathcal{X} \succeq Y \Rightarrow X \succeq Y \Rightarrow \max \mathcal{X} \succeq Y \text { and } \\
X \succeq \max \mathcal{Y} \Rightarrow X \succeq Y \Rightarrow X \succeq \min \mathcal{Y} . \square
\end{gathered}
$$

Next we investigate which of the properties of the binary relation $\succeq$ are also satisfied by its extensions $\succeq_{1}, \ldots, \succeq_{6}$. Obviously, when $\succeq$ is not reflexive (resp., antisymmetric, transitive), neither are its extensions $\succeq_{\mathrm{i}}, i=1, \ldots, 6$. Conversely, we can establish the following:

Proposition 5. Let $\succeq$ be a binary relation on random variables, and let $\succeq_{\mathrm{i}}, i=$ $1, \ldots, 6$ denote its extensions to sets of random variables, given by Definition??.

(a) If $\succeq$ is reflexive, so are $\succeq_{3}$, $\succeq_{4}$ and $\succeq_{6}$.

(b) If $\succeq$ is antisymmetric, so is $\succeq_{1}$.

(c) If $\succeq$ is transitive, so are $\succeq_{\mathrm{i}}$ for $i=1,2,3,5,6$.

Proof. First of all, if $\succeq$ is reflexive, $X \equiv X$ for any random variable $X$, and applying Definition ?? we deduce that $\mathcal{X} \succeq_{\mathrm{i}} \mathcal{X}$ for any $i=3,4,6$ and any set of random variables $\mathcal{X}$.

Secondly, assume that $\succeq$ is antisymmetric and that two sets of random variables $\mathcal{X}, \mathcal{Y}$ satisfy $\mathcal{X} \succeq_{1} \mathcal{Y}$ and $\mathcal{Y} \succeq_{1} \mathcal{X}$. Then, $X \succeq Y$ and $Y \succeq X$ for every $X \in \mathcal{X}$ and $Y \in \mathcal{Y}$, and by the antisymmetry property of $\succeq$, we deduce that $X=Y$ for every $X \in \mathcal{X}, Y \in \mathcal{Y}$. But this can only be if $\mathcal{X}=\{Z\}=\mathcal{Y}$ for some random variable $Z$. As a consequence, $\succeq_{1}$ is antisymmetric.

Finally, assume that $\succeq$ is transitive, and let us show that so are $\succeq_{i}$ for $i=1,2,3,5,6$. Consider three sets of random variables $\mathcal{X}, \mathcal{Y}, \mathcal{Z}$ :

1. If $\mathcal{X} \succeq_{1} \mathcal{Y}$ and $\mathcal{Y} \succeq_{1} \mathcal{Z}$ then $X \succeq Y$ and $Y \succeq Z$ for every $X \in \mathcal{X}, Y \in$ $\mathcal{Y}, Z \in \mathcal{Z}$. Applying the transitivity of $\succeq$, we deduce that $X \succeq Z$ for every $X \in \mathcal{X}, Z \in \mathcal{Z}$, and as a consequence $\mathcal{X} \succeq_{1} \mathcal{Z}$.

2. If $\mathcal{X} \succeq_{2} \mathcal{Y}$ and $\mathcal{Y} \succeq_{2} \mathcal{Z}$, there is $X \in \mathcal{X}$ such that $X \succeq Y$ for every $Y \in \mathcal{Y}$ and there is $Y^{*} \in \mathcal{Y}$ such that $Y^{*} \succeq Z$ for every $Z \in \mathcal{Z}$. In particular, $X \succeq Y^{*} \succeq Z$ for every $Z \in \mathcal{Z}$, whence, by the transitivity of $\succeq, \mathcal{X} \succeq_{2} \mathcal{Z}$.

3. If $\mathcal{X} \succeq_{3} \mathcal{Y}$ and $\mathcal{Y} \succeq_{3} \mathcal{Z}$, for every $Y \in \mathcal{Y}$ there is some $X_{Y} \in \mathcal{X}$ such that $X_{Y} \succeq Y$, and for every $Z \in \mathcal{Z}$ there is $Y_{Z} \in \mathcal{Y}$ such that $Y_{Z} \succeq Z$. As a consequence, for every $Z \in \mathcal{Z}$ it holds that $X_{Y_{Z}} \succeq Z$, and therefore $\mathcal{X} \succeq_{3} \mathcal{Z}$.

The transitivity of $\succeq_{5}, \succeq_{6}$ is proved similarly to that of $\succeq_{2}, \succeq_{3}$, respectively.

In addition, it is easy to check that reflexivity, antisymmetry and transitivity do not hold for definitions different than the ones of statements (a), (b) and (c), respectively.

Another interesting property in a binary relation is that it is complete, in the sense that given any two elements, either one is preferred to the other or 
they are indifferent, but they are never incomparable. From Proposition ??, it follows that the incomparable pairs with respect to an extension $\succeq_{i}$ are also incomparable with respect to the stronger extensions. The following result shows that if $\succeq$ is a complete relation, then its weakest extensions (namely, $\succeq_{3}, \succeq_{4}$ and $\succeq_{6}$ ) also induce complete binary relations:

Proposition 6. Let $\succeq$ be a binary relation on random variables, and let $\succeq_{\mathrm{i}}, i=$ $1, \ldots, 6$ be its extensions to sets of random variables given by Definition ??. If $\succeq$ is complete, then so are $\succeq_{3}, \succeq_{4}$ and $\succeq_{6}$.

Proof. Let $\mathcal{X}, \mathcal{Y}$ be two sets of random variables, and assume that $\mathcal{X} \nsucceq 3$ Y. Then there is some $Y \in \mathcal{Y}$ such that $X \nsucceq Y$ for all $X \in \mathcal{X}$. But since $\succeq$ is a complete relation, this means that $Y \succeq X$ for all $X \in \mathcal{X}$. As a consequence, $\mathcal{Y} \succeq_{2} \mathcal{X}$, and applying Proposition ?? we deduce that $\mathcal{Y} \succeq_{3} \mathcal{X}$. Hence, the binary relation $\succeq_{3}$ is complete.

On the other hand, if $\mathcal{X} \nsucceq_{4} \mathcal{Y}$, we deduce from Proposition ?? that also $\mathcal{X} \nsucceq_{3} \mathcal{Y}$, whence the above reasoning implies that $\mathcal{Y} \succeq_{3} \mathcal{X}$ and again from Proposition ?? we deduce that $\mathcal{Y} \succeq_{4} \mathcal{X}$.

The proof that $\succeq_{6}$ also induces a complete relation is analogous.

Remark 3. Although in this paper we shall focus on the particular application of Definition ?? to the relation $\succeq$ associated to stochastic dominance or statistical preference, there are other cases of interest. Perhaps the most important one is that where the comparison between pairs of random variables is made by means of their expected utility:

$$
X \succeq Y \Leftrightarrow E(X) \geq E(Y) ;
$$

it is not difficult to see that Definition ?? gives rise to some well-known generalizations of expected utility that are formulated in terms of lower and upper expectations. Consider two sets $\mathcal{X}, \mathcal{Y}$ and assume that the expectations of all their elements exist. Then with respect to definition $\succeq_{1}$ it holds that:

$$
\mathcal{X} \succeq_{1} \mathcal{Y} \Leftrightarrow \underline{E}(\mathcal{X})=\inf _{X \in \mathcal{X}} E(X) \geq \sup _{Y \in \mathcal{Y}} E(Y)=\bar{E}(\mathcal{Y}),
$$

which relates this notion to the concept of interval dominance in [?].

If we now consider definition $\succeq_{3}$, it holds that

$$
\mathcal{X} \succeq_{3} \mathcal{Y} \Rightarrow \bar{E}(\mathcal{X})=\sup _{X \in \mathcal{X}} E(X) \geq \sup _{Y \in \mathcal{Y}} E(Y)=\bar{E}(\mathcal{Y}) .
$$

Thus, definition $\succeq_{3}$ is stronger than the maximax criterium [?], which is based on comparing the best possibilities in our sets of alternatives. Similarly, if we consider definition $\succeq_{6}$ it holds that:

$$
\mathcal{X} \succeq_{6} \mathcal{Y} \Rightarrow \underline{E}(\mathcal{X})=\inf _{X \in \mathcal{X}} E(X) \geq \inf _{Y \in \mathcal{Y}} E(Y)=\underline{E}(\mathcal{Y}) .
$$

Thus, definition $\succeq_{6}$ is stronger than the maximin criterium [? ], which compares the worst possibilities within the sets of alternatives. 
Finally, definition $\succeq_{4}$ implies that

$$
\mathcal{X} \succeq_{4} \mathcal{Y} \Rightarrow \bar{E}(\mathcal{X})=\sup _{X \in \mathcal{X}} E(X) \geq \inf _{Y \in \mathcal{Y}} E(Y)=\underline{E}(\mathcal{Y}),
$$

so if $\mathcal{X}$ is $\succeq_{4}$-preferred to $\mathcal{Y}$ then it is also preferred with respect to the criterion of E-admissibility from [? ]. See [? ? ] for related comments.

\subsection{Imprecise Stochastic Dominance}

In this subsection, we explore in some detail the case where the binary relation $\succeq$ is the one associated to the notion of stochastic dominance we have introduced in Definition ??, i.e., the relation $\succeq$ is defined by $\succeq_{\text {FSD }}$. We shall assume that the utility space $\Omega^{\prime}$ is $[0,1]$, although the results can be immediately extended to any bounded interval of real numbers.

From [? , Example 1], we can see that the converse implications in Proposition ?? do not hold in general. With respect to the other results, since the relation $\succeq_{\text {FSD }}$ is reflexive and transitive, we can apply Proposition ?? and characterize the equivalences between $\succeq_{\mathrm{FSD}_{2}}$ and $\succeq_{\mathrm{FSD}_{3}}$, and also between $\succeq_{\mathrm{FSD}_{5}}$ and $\succeq_{\mathrm{FSD}_{6}}$ by means of the existence of a maximum and a minimum value in the sets $\mathcal{X}, \mathcal{Y}$ we want to compare. Moreover, we can deduce from Proposition ?? that $\succeq_{\mathrm{FSD}_{\mathrm{i}}}$ is reflexive for $i=3,4,6$ and transitive for $i=1,2,3,5,6$. On the other hand, since two different random variables may induce the same distribution function, $\succeq_{\text {FSD }}$ is not antisymmetric.

Since $\succeq_{\text {FSD }}$ also complies with Pareto dominance (Eq. (??)), we deduce from Proposition ?? that when the sets $\mathcal{X}, \mathcal{Y}$ to compare have both a maximum and a minimum element, we can easily characterize the conditions $\succeq_{\mathrm{FSD}_{\mathrm{i}}}, i=1, \ldots, 6$ by comparing these maximum and minimum elements only.

Finally, note that $\succeq_{\text {FSD }}$ is not a complete relation, because there are distribution functions $F, G$ such that $F \nsucceq_{\mathrm{FSD}} G$ and $G \succeq_{\mathrm{FSD}} F$. As a consequence, Proposition ?? is not applicable in this context.

The relations $\succeq_{\mathrm{FSD}_{\mathrm{i}}}, i=1, \ldots, 6$, were already studied in [? ] as a generalization of stochastic dominance towards sets of alternatives. Since stochastic dominance compares the alternatives by means of their associated distribution functions, this extension to an imprecise context may be made in terms of the comparison of sets of distribution functions, which can be equivalently represented by means of their associated p-boxes. We have proved the following:

Proposition 7. [? , Proposition 3] Let $\mathcal{F}_{\mathrm{X}}$ and $\mathcal{F}_{\mathrm{Y}}$ be two sets of cumulative distribution functions, and denote by $\left(\underline{F}_{\mathrm{X}}, \bar{F}_{\mathrm{X}}\right)$ and $\left(\underline{F}_{\mathrm{Y}}, \bar{F}_{\mathrm{Y}}\right)$ the p-boxes they induce by means of Eq. (??). Then the following statements hold:

1. $\mathcal{F}_{\mathrm{X}} \succeq_{\mathrm{FSD}_{1}} \mathcal{F}_{\mathrm{Y}} \Leftrightarrow \bar{F}_{\mathrm{X}} \succeq_{\mathrm{FSD}} \underline{F}_{\mathrm{Y}}$.

2. $\mathcal{F}_{\mathrm{X}} \succeq_{\mathrm{FSD}_{2}} \mathcal{F}_{\mathrm{Y}} \Rightarrow \underline{F}_{\mathrm{X}} \succeq_{\mathrm{FSD}} \underline{F}_{\mathrm{Y}}$.

3. $\mathcal{F}_{\mathrm{X}} \succeq_{\mathrm{FSD}_{3}} \mathcal{F}_{\mathrm{Y}} \Rightarrow \underline{F}_{\mathrm{X}} \succeq_{\mathrm{FSD}} \underline{F}_{\mathrm{Y}}$.

4. $\mathcal{F}_{\mathrm{X}} \succeq_{\mathrm{FSD}_{4}} \mathcal{F}_{\mathrm{Y}} \Rightarrow \underline{F}_{\mathrm{X}} \succeq_{\mathrm{FSD}} \bar{F}_{\mathrm{Y}}$.

5. $\mathcal{F}_{\mathrm{X}} \succeq_{\mathrm{FSD}_{5}} \mathcal{F}_{\mathrm{Y}} \Rightarrow \bar{F}_{\mathrm{X}} \succeq_{\mathrm{FSD}} \bar{F}_{\mathrm{Y}}$.

6. $\mathcal{F}_{\mathrm{X}} \succeq_{\mathrm{FSD}_{6}} \mathcal{F}_{\mathrm{Y}} \Rightarrow \bar{F}_{\mathrm{X}} \succeq_{\mathrm{FSD}} \bar{F}_{\mathrm{Y}}$. 
Moreover, when both the lower and the upper distribution functions belong to the respective sets, the converse implications also hold.

Note that the second part of this result can be seen now as a consequence of Propositions ?? and ??.

Next, we provide an alternative characterization of imprecise stochastic dominance in terms of the equivalent formulation given in Theorem ??. Recall that given two random variables $X$ and $Y$, it holds that $X \succeq_{\text {FSD }} Y$ if and only if $E(u(X)) \geq E(u(Y))$ for every non-decreasing function $u$. When we compare sets of random variables, we must replace these expectations by lower and upper expectations. For any given set of distribution functions $\mathcal{F}$ and any nondecreasing function $u:[0,1] \rightarrow \mathbb{R}$, let us denote $\underline{E}_{\mathcal{F}}(u):=\inf _{F \in \mathcal{F}} E_{P_{F}}(u)$ and $\bar{E}_{\mathcal{F}}(u):=\sup _{F \in \mathcal{F}} E_{P_{F}}(u)$. It is easy to establish the following result:

Theorem 8. Let us consider two sets of cumulative distribution functions $\mathcal{F}_{1}$ and $\mathcal{F}_{2}$, and let $\mathcal{U}$ be the set of all non-decreasing functions $u:[0,1] \rightarrow \mathbb{R}$. The following statements hold:

1. $\mathcal{F}_{1} \succeq_{\mathrm{FSD}_{1}} \mathcal{F}_{2} \Leftrightarrow \underline{E}_{\mathcal{F}_{1}}(u) \geq \bar{E}_{\mathcal{F}_{2}}(u)$ for every $u \in \mathcal{U}$.

2. $\mathcal{F}_{1} \succeq_{\mathrm{FSD}_{2}} \mathcal{F}_{2} \Rightarrow \bar{E}_{\mathcal{F}_{1}}(u) \geq \bar{E}_{\mathcal{F}_{2}}(u)$ for every $u \in \mathcal{U}$.

3. $\mathcal{F}_{1} \succeq_{\mathrm{FSD}_{3}} \mathcal{F}_{2} \Rightarrow \bar{E}_{\mathcal{F}_{1}}(u) \geq \bar{E}_{\mathcal{F}_{2}}(u)$ for every $u \in \mathcal{U}$.

4. $\mathcal{F}_{1} \succeq_{\mathrm{FSD}_{4}} \mathcal{F}_{2} \Rightarrow \bar{E}_{\mathcal{F}_{1}}(u) \geq \underline{E}_{\mathcal{F}_{2}}(u)$ for every $u \in \mathcal{U}$.

5. $\mathcal{F}_{1} \succeq_{\mathrm{FSD}_{5}} \mathcal{F}_{2} \Rightarrow \underline{E}_{\mathcal{F}_{1}}(u) \geq \underline{E}_{\mathcal{F}_{2}}(u)$ for every $u \in \mathcal{U}$.

6. $\mathcal{F}_{1} \succeq_{\mathrm{FSD}_{6}} \mathcal{F}_{2} \Rightarrow \underline{E}_{\mathcal{F}_{1}}(u) \geq \underline{E}_{\mathcal{F}_{2}}(u)$ for every $u \in \mathcal{U}$.

Taking into account Eq. (??), the above implications hold in particular when we replace the set $\mathcal{U}$ by the subset $\mathcal{U}^{*}$ of the non-decreasing and bounded maps $u: \mathbb{R} \rightarrow \mathbb{R}$. This will be useful when comparing random sets by means of stochastic dominance in Section ??.

\subsection{Imprecise Statistical Preference}

We consider next the case where the basic binary relation to generalize is that associated to statistical preference, as given in Definition ??. Hence, we shall assume in general that the utility space $\Omega^{\prime}$ is an ordered set, which need not be numerical.

Let us denote by $\succeq_{\mathrm{SP}_{\mathrm{i}}}, i=1, \ldots, 6$ the conditions obtained by means of Definition ??. Note that $\succeq_{\mathrm{SP}}$ is reflexive and complete, but it is neither antisymmetric not transitive. Hence, Proposition ?? does not apply in this case; indeed, we can use statistical preference to show that Proposition ?? cannot be extended to non transitive relationships:

Example 2. Consider the random variables $X, Y, Z$ from Example ?? such that $X \succ_{\mathrm{SP}} Y \succ_{\mathrm{SP}} Z \succ_{\mathrm{SP}} X$, and let $\mathcal{X}=\{X, Y\}, \mathcal{Y}=\{X, Z\}$. Then since $X \succeq_{\mathrm{SP}} X$ and $Y \succeq_{\mathrm{SP}} Z$, we deduce that $\mathcal{X} \succeq_{\mathrm{SP}_{3}} \mathcal{Y}$; since $X \succ_{\mathrm{SP}} Y$ and $Z \succ_{\mathrm{SP}} X$, we see that $\mathcal{X} \nsucceq_{\mathrm{SP}_{2}} \mathcal{Y}$; however, $\mathcal{X}$ has a maximum element, because $X \succ_{\mathrm{SP}} Y$. 
On the other hand, since statistical preference complies with Pareto dominance we deduce from Proposition ?? that the different conditions can be reduced to the comparison of the maximum and minimum elements of $\mathcal{X}, \mathcal{Y}$, when these maximum and minimum elements exist. Finally, we deduce from Propositions ?? and ?? that conditions $\succeq_{\mathrm{SP}_{3}}, \succeq_{\mathrm{SP}_{4}}, \succeq_{\mathrm{SP}_{6}}$ induce a reflexive and complete relationship.

We can also use statistical preference to show that Proposition ?? cannot be extended to the relations $\succeq_{1}, \succeq_{2}$ nor $\succeq_{5}$ : take the sets $\mathcal{X}=\mathcal{Y}=\{X, Y, Z\}$, where the variables $X, Y, Z$ satisfy $X \succ_{\mathrm{SP}} Y \succ_{\mathrm{SP}} Z \succ_{\mathrm{SP}} X$ as in Example ??; then the set $\mathcal{X}$ has neither a maximum nor a minimum element, whence it is incomparable with itself with respect to $\succeq_{\mathrm{SP}_{2}}$ and $\succeq_{\mathrm{SP}_{5}}$. Applying Proposition ??, we deduce that $\mathcal{X}, \mathcal{Y}$ are also incomparable with respect to $\succeq \mathrm{SP}_{1}$.

We showed in Theorem ?? that the generalizations of stochastic dominance towards sets of variables are related to lower and upper expectations. Next, we establish a similar result for the generalizations of statistical preference. The result shall be established in terms of lower and upper medians, and for this we shall require that our utility space $\Omega^{\prime}$ is numerical. Let us consider two sets of alternatives $\mathcal{X}, \mathcal{Y}$ with values on $\Omega^{\prime}$, and let us introduce the following notation:

$$
\begin{aligned}
& \operatorname{Me}(\mathcal{X}-\mathcal{Y})=\{\operatorname{Me}(X-Y): X \in \mathcal{X}, Y \in \mathcal{Y}\} \\
& \overline{\operatorname{Me}}(\mathcal{X}-\mathcal{Y})=\inf \operatorname{Me}(\mathcal{X}-\mathcal{Y}) \\
& \overline{\operatorname{Me}}(\mathcal{X}-\mathcal{Y})=\sup \operatorname{Me}(\mathcal{X}-\mathcal{Y})
\end{aligned}
$$

where the median of a random variable with respect to a probability measure is given by $M e_{\mathrm{P}}(X):=\inf \{t: P(X \leq t) \geq 0.5\}$.

Proposition 9. Let $\mathcal{X}, \mathcal{Y}$ be two sets of random variables defined on a probability space $(\Omega, \mathcal{A}, P)$ and taking values on $\mathbb{R}$.

1. $\underline{\mathrm{Me}}(\mathcal{X}-\mathcal{Y})>0 \Rightarrow \mathcal{X} \succeq_{\mathrm{SP}_{1}} \mathcal{Y} \Rightarrow \underline{\mathrm{Me}}(\mathcal{X}-\mathcal{Y}) \geq 0$

2. $\exists X \in \mathcal{X}$ s.t. $\underline{\operatorname{Me}}(\{X\}-\mathcal{Y})>0 \Rightarrow \mathcal{X} \succeq_{\mathrm{SP}_{2}} \mathcal{Y} \Rightarrow \exists X \in \mathcal{X}$ s.t. $\underline{\operatorname{Me}}(\{X\}-$ $\mathcal{Y}) \geq 0$.

3. $\overline{\operatorname{Me}}(\mathcal{X}-\{Y\})>0 \forall Y \in \mathcal{Y} \Rightarrow \mathcal{X} \succeq_{\mathrm{SP}_{3}} \mathcal{Y} \Rightarrow \overline{\operatorname{Me}}(\mathcal{X}-\{Y\}) \geq 0 \forall Y \in \mathcal{Y}$.

4. $\overline{\mathrm{Me}}(\mathcal{X}-\mathcal{Y})>0 \Rightarrow \mathcal{X} \succeq_{\mathrm{SP}_{4}} \mathcal{Y} \Rightarrow \overline{\mathrm{Me}}(\mathcal{X}-\mathcal{Y}) \geq 0$.

5. $\exists Y \in \mathcal{Y}$ s.t. $\underline{\operatorname{Me}}(\mathcal{X}-\{Y\})>0 \Rightarrow \mathcal{X} \succeq_{\mathrm{SP}_{5}} \mathcal{Y} \Rightarrow \exists Y \in \mathcal{Y}$ s.t. $\underline{\operatorname{Me}}(\mathcal{X}-$ $\{Y\}) \geq 0$.

6. $\overline{\operatorname{Me}}(\{X\}-\mathcal{Y})>0 \forall X \in \mathcal{X} \Rightarrow \mathcal{X} \succeq_{\mathrm{SP}_{6}} \mathcal{Y} \Rightarrow \overline{\operatorname{Me}}(\{X\}-\mathcal{Y}) \geq 0 \forall X \in \mathcal{X}$.

Proof. From [?, Theorem 5], given two random variables $X, Y$,

$$
M e(X-Y)>0 \Rightarrow X \succeq_{\mathrm{SP}} Y \Rightarrow M e(X-Y) \geq 0 .
$$

The result follows from this equation together with Definition ?? and the above definitions of lower and upper median.

Taking into account the properties of the median, we conclude from this result that statistical preference may be seen as a more robust alternative to stochastic dominance or expected utility in the presence of outliers. 


\subsubsection{Connection with aggregation operators}

Since the binary relation associated to statistical preference is complete, we deduce from Proposition ?? that so are the relations $\succeq_{\mathrm{SP}_{3}}, \succeq_{\mathrm{SP}_{4}}, \succeq_{\mathrm{SP}_{6}}$. Complete relations are interesting because they mean that we can always express a preference between two sets of alternatives $\mathcal{X}, \mathcal{Y}$. Another way of deriving a complete relation when we make multiple comparisons is to establish a degree of preference for every pairwise comparison, and to aggregate these degrees of preference into a joint one. This is possible to do by means of an aggregation operator.

Let $\mathcal{X}=\left\{X_{1}, \ldots, X_{n}\right\}$ and $\mathcal{Y}=\left\{Y_{1}, \ldots, Y_{m}\right\}$ be two finite sets of random variables taking values on an ordered utility space $\Omega^{\prime}$, and let us compute the statistical preference $Q\left(X_{i}, Y_{j}\right)$ for every pair of variables $X_{i} \in \mathcal{X}, Y_{j} \in \mathcal{Y}$ by means of Eq. (??). The set of all these preferences is an instance of profile of preferences [?], and can be represented by means of the matrix

$$
\mathbb{Q} \mathcal{X}, \mathcal{Y}:=\left(\begin{array}{cccc}
Q\left(X_{1}, Y_{1}\right) & Q\left(X_{1}, Y_{2}\right) & \ldots & Q\left(X_{1}, Y_{m}\right) \\
\vdots & \vdots & \vdots & \vdots \\
Q\left(X_{n}, Y_{1}\right) & Q\left(X_{n}, Y_{2}\right) & \ldots & Q\left(X_{n}, Y_{m}\right)
\end{array}\right)
$$

Note that the profile of preferences of $\mathcal{Y}$ over $\mathcal{X}, \mathbb{Q} y, \mathcal{X}$, corresponds to one minus the transposed matrix of $\mathbb{Q} \mathcal{X}, \mathcal{Y}$, i.e., $1-\mathbb{Q}_{\mathcal{X}, \mathcal{Y}}^{t}$. We shall show that conditions $\succeq_{\mathrm{SP}_{1}}, \ldots, \succeq_{\mathrm{SP}_{6}}$ can be expressed by means of an aggregation operator over the profile of preference:

Definition 6. [? ? ] An aggregation function is a mapping $F: \cup_{s \in \mathbb{N}}[0,1]^{s} \rightarrow$ $[0,1]$. It is called an aggregation operator when it satisfies the following conditions:

- $x_{i} \leq y_{i} \forall i=1, \ldots, s \Rightarrow F\left(x_{1}, \ldots, x_{s}\right) \leq F\left(y_{1}, \ldots, y_{s}\right) \cdot$ [Monotonicity]

- $F(0, \ldots, 0)=0$ and $F(1, \ldots, 1)=1$. [Boundary conditions]

The matrix $\mathbb{Q}_{\mathcal{X}, \mathcal{Y}}$ representing the profile of preferences between $\mathcal{X}$ and $\mathcal{Y}$ can be equivalently represented by means of a vector on $[0,1]^{n m}$ using the lexicographic order:

$$
\vec{z}_{\mathcal{X}, \mathcal{Y}}=\left(Q\left(X_{1}, Y_{1}\right), Q\left(X_{1}, Y_{2}\right), \ldots, Q\left(X_{1}, Y_{m}\right), Q\left(X_{2}, Y_{1}\right), \ldots, Q\left(X_{n}, Y_{m}\right)\right) .
$$

Taking this into account, given an aggregation function $F: \cup_{s \in \mathbb{N}}[0,1]^{s} \rightarrow[0,1]$, we shall denote by $F(\mathbb{Q} \mathcal{X}, \mathcal{Y})$ the image it gives to the vector $\vec{z}_{\mathcal{X}, \mathcal{Y}}$.

Definition 7. Given two finite sets of random variables $\mathcal{X}=\left\{X_{1}, \ldots, X_{n}\right\}$ and $\mathcal{Y}=\left\{Y_{1}, \ldots, Y_{m}\right\}$ and an aggregation function $F$, we say that $\mathcal{X}$ is $F$ statistically preferred to $\mathcal{Y}$, and denote it by $\mathcal{X} \succeq_{\operatorname{SP}_{F}} \mathcal{Y}$, if

$$
F\left(\mathbb{Q}_{\mathcal{X}, \mathcal{Y}}\right):=F\left(\vec{z}_{\mathcal{X}, \mathcal{Y}}\right) \geq \frac{1}{2} .
$$


We refer to [?] for a review of aggregation operators. Some important properties are the following:

Definition 8. [? ] An aggregation function $F: \cup_{s \in \mathbb{N}}[0,1]^{s} \rightarrow[0,1]$ is called:

- symmetric if it is invariant under permutations.

- monotone if $F\left(r_{1}, \ldots, r_{s}\right) \geq F\left(r_{1}^{\prime}, \ldots, r_{s}^{\prime}\right)$ whenever $r_{i} \geq r_{i}^{\prime}$ for every $i=$ $1, \ldots, s$.

- idempotent if $F(r, \ldots, r)=r$.

We shall call an aggregation function $F: \cup_{s \in \mathbb{N}}[0,1]^{s} \rightarrow[0,1]$ self-dual if $F\left(r_{1}, \ldots, r_{s}\right)=1-F\left(1-r_{1}, \ldots, 1-r_{s}\right)$ for every $\left(r_{1}, \ldots, r_{s}\right) \in[0,1]^{s}$ and for every $s \in \mathbb{N}$.

All these properties are interesting when aggregating the profile of preferences into a joint one: symmetry implies that all the elements in the profile are given the same weight; idempotence means that if all the preference degrees equal $r$, the final preference degree should also equal $r$; monotonicity assures that if we increase all the values in the profile of preferences, the final value should also increase; and self-duality preserves the idea behind the notion of probabilistic relation in Definition ??, since for a self-dual aggregation function $F, F\left(\mathbb{Q}_{\mathcal{X}, \mathcal{Y}}^{t}\right)+F\left(\mathbb{Q}_{\mathcal{Y}, \mathcal{X}}\right)=1$. If in addition $F$ is symmetric, we obtain that $F(\mathbb{Q} \mathcal{X}, \mathcal{Y})+F(\mathbb{Q} \mathcal{Y}, \mathcal{X})=1$. This last property means that, when $F$ is a self-dual and symmetric aggregation function, Eq. (??) is equivalent to $F\left(\mathbb{Q}_{\mathcal{X}, \mathcal{Y}}\right) \geq F\left(\mathbb{Q}_{\mathcal{Y}, \mathcal{X}}\right)$

The relations $\succeq_{\mathrm{SP}_{\mathrm{i}}}$, for $i=1, \ldots, 6$, can all expressed by means of an aggregation function, as we summarize in the following proposition. Its proof is immediate and therefore omitted.

Proposition 10. Let $\mathcal{X}=\left\{X_{1}, \ldots, X_{n}\right\}, \mathcal{Y}=\left\{Y_{1}, \ldots, Y_{m}\right\}$ be two finite sets of random variables taking values on an ordered space $\Omega^{\prime}$. Then for any $i=1, \ldots, 6$ $\mathcal{X} \succeq_{\mathrm{SP}_{\mathrm{i}}} \mathcal{Y}$ if and only if it is $F_{i}$-statistically preferred to $\mathcal{Y}$, where the aggregation functions $F_{i}$ are given by:

$$
\begin{aligned}
& F_{1}(\mathbb{Q} \mathcal{X}, \mathcal{Y}):=\min _{i, j} Q\left(X_{i}, Y_{j}\right) . \\
& F_{2}(\mathbb{Q} \mathcal{X}, \mathcal{Y}):=\max _{i=1, \ldots, n} \min _{j=1, \ldots, m} Q\left(X_{i}, Y_{j}\right) . \\
& F_{3}(\mathbb{Q} \mathcal{X}, \mathcal{Y}):=\min _{j=1, \ldots, m} \max _{i=1, \ldots, n} Q\left(X_{i}, Y_{j}\right) . \\
& F_{4}\left(\mathbb{Q}_{\mathcal{X}, \mathcal{Y}}\right):=\max _{i, j} Q\left(X_{i}, Y_{j}\right) . \\
& F_{5}(\mathbb{Q} \mathcal{X}, \mathcal{Y}):=\max _{j=1, \ldots, m} \min _{i=1, \ldots, n} Q\left(X_{i}, Y_{j}\right) . \\
& F_{6}\left(\mathbb{Q}_{\mathcal{X}, \mathcal{Y}}\right):=\min _{i=1, \ldots, n} \max _{j=1, \ldots, m} Q\left(X_{i}, Y_{j}\right) .
\end{aligned}
$$

It is not difficult to see that all the aggregation functions $F_{i}$ above are monotonic and comply with the boundary conditions $F_{i}(0, \ldots, 0)=0$ and $F_{i}(1, \ldots, 1)=1$; 
they are thus instances of aggregation operators. They are moreover idempotent. On the other hand, only $F_{1}$ and $F_{4}$ are symmetric, and none of them is self-dual.

We can also use these aggregation operators to deduce the relationships between the different conditions established in Proposition ?? in the case of statistical preference: it suffices to take into account that $F_{1} \leq F_{2} \leq F_{3} \leq F_{4}$ and $F_{1} \leq F_{5} \leq F_{6} \leq F_{4}$.

The above remarks suggest that other preference relationships may be defined by means of other aggregation operators $F$, and this would allow us to take all the elements of the profile of preferences into account, instead of focusing on the best or worst scenarios only. Next, we explore briefly one of these possibilities: the arithmetic mean $F_{\text {mean }}$, given by

$$
\begin{aligned}
F_{\text {mean }}: \cup_{s \in \mathbb{N}}[0,1]^{s} & \rightarrow[0,1] \\
\left(r_{1}, \ldots, r_{s}\right) & \hookrightarrow \frac{r_{1}+\cdots+r_{s}}{s} .
\end{aligned}
$$

This is a symmetric, monotone, idempotent and self-dual aggregation opera-

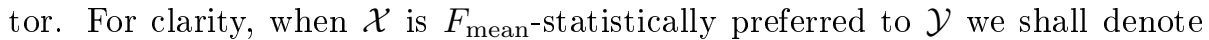
it $\mathcal{X} \succeq_{\mathrm{SP}_{\text {mean }}} \mathcal{Y}$. The connection between $\succeq_{\mathrm{SP}_{\text {mean }}}$ and $\succeq_{\mathrm{SP}_{\mathrm{i}}}, i=1, \ldots, 6$ is a consequence of the following result:

Proposition 11. Given two finite sets of random variables $\mathcal{X}=\left\{X_{1}, \ldots, X_{n}\right\}$ and $\mathcal{Y}=\left\{Y_{1}, \ldots, Y_{m}\right\}$ and a monotone and idempotent aggregation function $F$,

$$
\mathcal{X} \succeq_{\mathrm{SP}_{1}} \mathcal{Y} \Rightarrow \mathcal{X} \succeq_{\mathrm{SP}_{\mathrm{F}}} \mathcal{Y} \Rightarrow \mathcal{X} \succeq_{\mathrm{SP}_{4}} \mathcal{Y}
$$

Proof. On the one hand, assume that $\mathcal{X} \succeq_{\mathrm{SP}_{1}} \mathcal{Y}$. Then, $Q(X, Y) \geq \frac{1}{2}$ for every $X \in \mathcal{X}$ and $Y \in \mathcal{Y}$. Since $F$ is monotone and idempotent, $F\left(\mathbb{Q}_{\mathcal{X}}, \mathcal{Y}\right) \geq$ $F\left(\frac{1}{2}, \ldots, \frac{1}{2}\right)=\frac{1}{2}$, and consequently $\mathcal{X} \succeq_{\mathrm{SP}_{\mathrm{F}}} \mathcal{Y}$.

On the other hand, assume ex-absurdo that $F\left(\mathbb{Q}_{\mathcal{X}, \mathcal{Y}}\right) \geq \frac{1}{2}$ and that $\mathcal{X} \nsucceq_{\mathrm{SP}_{4}}$ $\mathcal{Y}$, so that $Q(X, Y)<\frac{1}{2}$ for every $X \in \mathcal{X}$ and $Y \in \mathcal{Y}$. Then $F\left(\mathbb{Q}_{\mathcal{X}, \mathcal{Y}}\right) \leq$ $\max _{i, j} Q\left(X_{i}, Y_{j}\right)<\frac{1}{2}$, a contradiction. Hence, $\mathcal{X} \succeq_{\mathrm{SP}_{4}} \mathcal{Y}$.

In particular, we see that $\succeq_{\mathrm{SP}_{\text {mean }}}$ is an intermediate notion between $\succeq_{\mathrm{SP}_{1}}$ and $\succeq_{\mathrm{SP}_{4}}$. To see that it is not related to $\succeq_{\mathrm{SP}_{\mathrm{i}}}$ for $i=2,3,5,6$, consider the following example:

Example 3. Consider $\Omega=\left\{\omega_{1}, \omega_{2}\right\}\left(P\left(\left\{\omega_{i}\right\}\right)=1 / 2\right)$, and the sets of random variables $\mathcal{X}=\left\{X_{1}, X_{2}, X_{3}\right\}$ and $\mathcal{Y}=\{Y\}$ defined by:

\begin{tabular}{c|cc} 
& $\omega_{1}$ & $\omega_{2}$ \\
\hline$X_{1}$ & 0 & 2 \\
$X_{2}$ & 0 & 0 \\
$X_{3}$ & 2 & 2 \\
$Y$ & 1 & 1
\end{tabular}

Then,

$$
\mathbb{Q}_{\mathcal{X}, \mathcal{Y}}:=\left(\begin{array}{c}
\frac{1}{2} \\
0 \\
1
\end{array}\right) \text { and } \mathbb{Q} \mathcal{Y}, \mathcal{X}:=\left(\begin{array}{ccc}
\frac{1}{2} & 1 & 0
\end{array}\right)
$$


whence by Proposition ?? $\mathcal{X} \succ_{\mathrm{SP}_{\mathrm{i}}} \mathcal{Y}$, for $i=2,3$, and $\mathcal{Y} \succ_{\mathrm{SP}_{\mathrm{i}}} \mathcal{X}$, for $i=5,6$. On the other hand,

$$
\frac{Q\left(X_{1}, Y\right)+Q\left(X_{2}, Y\right)+Q\left(X_{3}, Y\right)}{3}=\frac{1}{2},
$$

and consequently $\mathcal{X} \equiv_{\mathrm{SP}_{\text {mean }}} \mathcal{Y}$. Hence, $\mathcal{X} \succeq_{\mathrm{SP}_{\text {mean }}} \mathcal{Y} \nRightarrow \mathcal{X} \succeq_{\mathrm{SP}_{\mathrm{i}}} \mathcal{Y}$ for $i=5,6$, and $\mathcal{Y} \succeq_{\mathrm{SP}_{\text {mean }}} \mathcal{X} \nRightarrow \mathcal{Y} \succeq_{\mathrm{SP}_{\mathrm{i}}} \mathcal{X}$ for $i=2,3$. By comparing $\mathcal{Z}_{1}=\left\{X_{2}, Y\right\}$ and $\mathcal{Z}_{2}=\left\{X_{3}, Y\right\}$ with $\mathcal{X}$, we can see that the converse implications do not hold: $\mathcal{Z}_{1} \equiv_{\mathrm{SP}_{5,6}} \mathcal{X} \succ_{\mathrm{SP}_{\text {mean }}} \mathcal{Z}_{1}$ and $\mathcal{Z}_{2} \succ_{\mathrm{SP}_{\text {mean }}} \mathcal{X} \equiv_{\mathrm{SP}_{2,3}} \mathcal{Z}_{2} \downarrow$

\section{Comparison of random sets}

In the next two sections, we shall show how the above results can be applied in two different scenarios of imprecision within a decision problem: the case where we have imprecise information about the utilities of the different alternatives, and that where we have imprecise beliefs about the states of nature.

Let us start with the first case. Consider a decision problem where we must choose between two alternatives $X$ and $Y$ whose respective utilities depend on the values $\omega$ of the states of nature. Assume that we have precise information about the probabilities of these states of nature, so that $X$ and $Y$ can be seen as random variables defined on a probability space $(\Omega, \mathcal{A}, P)$. If we have imprecise knowledge about the utilities $X(\omega)$ associated to the different states of nature, one possible model would be to associate to any $\omega \in \Omega$ a set $\Gamma(\omega)$ which is sure to include the 'true' utility $X(\omega)$. By doing this, we obtain a multi-valued mapping $\Gamma: \Omega \rightarrow \mathcal{P}\left(\Omega^{\prime}\right)$, and all we know about $X$ is that it is one of the measurable selections of $\Omega$, i.e., that it belongs to the set

$$
S(\Gamma)=\left\{U: \Omega \rightarrow \Omega^{\prime} \text { r.v. }: U(\omega) \in \Gamma(\omega) \text { for every } \omega \in \Omega\right\} .
$$

This interpretation of multi-valued mappings, as a model for the imprecise knowledge of a random variable is not new, and can be traced back to Kruse and Meyer [? ]. This epistemic interpretation contrasts with the ontic interpretation which is sometimes given to random sets as naturally imprecise quantities [?].

In this paper, we shall consider only multi-valued mappings satisfying a certain measurability condition:

Definition 9. Let $(\Omega, \mathcal{A}, P)$ be a probability space, $\left(\Omega^{\prime}, \mathcal{A}^{\prime}\right)$ a measurable space and $\Gamma: \Omega \rightarrow \mathcal{P}\left(\Omega^{\prime}\right)$ a multi-valued mapping. It is called a random set when $\Gamma^{*}(A):=\{\omega \in \Omega: \Gamma(\omega) \cap A \neq \emptyset\} \in \mathcal{A} \forall A \in \mathcal{A}^{\prime}$.

Our comparison of two alternatives with imprecise utilities results thus in the comparison of two random sets $\Gamma_{1}, \Gamma_{2}$, that we shall compare by means of their respective sets of measurable selections $S\left(\Gamma_{1}\right), S\left(\Gamma_{2}\right)$ determined by Eq. (??). For simplicity, we shall use the notation $\Gamma_{1} \succeq \Gamma_{2}$ instead of $S\left(\Gamma_{1}\right) \succeq S\left(\Gamma_{2}\right)$ when no confusion is possible.

Let us begin by studying the comparison of random sets by means of stochastic dominance. 
Proposition 12. Let $(\Omega, \mathcal{A}, P)$ be a probability space, $\left(\Omega^{\prime}, \mathcal{P}\left(\Omega^{\prime}\right)\right)$ a measurable space, with $\Omega^{\prime}$ a finite subset of $\mathbb{R}$, and $\Gamma_{1}, \Gamma_{2}$ be two random sets. The following equivalences hold:

(a) $\Gamma_{1} \succeq_{\mathrm{FSD}_{1}} \Gamma_{2} \Leftrightarrow \min \Gamma_{1} \succeq_{\mathrm{FSD}} \max \Gamma_{2}$.

(b) $\Gamma_{1} \succeq_{\mathrm{FSD}_{2}} \Gamma_{2} \Leftrightarrow \Gamma_{1} \succeq_{\mathrm{FSD}_{3}} \Gamma_{2} \Leftrightarrow \max \Gamma_{1} \succeq_{\mathrm{FSD}} \max \Gamma_{2}$.

(c) $\Gamma_{1} \succeq_{\mathrm{FSD}_{4}} \Gamma_{2} \Leftrightarrow \max \Gamma_{1} \succeq_{\mathrm{FSD}} \min \Gamma_{2}$.

(d) $\Gamma_{1} \succeq_{\mathrm{FSD}_{5}} \Gamma_{2} \Leftrightarrow \Gamma_{1} \succeq_{\mathrm{FSD}_{6}} \Gamma_{2} \Leftrightarrow \min \Gamma_{1} \succeq_{\mathrm{FSD}} \min \Gamma_{2}$.

Proof. The result follows from Proposition ??, taking into account that given a random set $\Gamma$ taking values on a finite space, the lower distribution function associated to its set $S(\Gamma)$ of measurable selections is induced by $\max \Gamma$ and its upper distribution function is induced by $\min \Gamma$.

Moreover, we can characterize the conditions $\succeq_{\mathrm{FSD}_{\mathrm{i}}}, i=1, \ldots, 6$ even for random sets that take values on infinite spaces. To see how this comes out, we must recall first the notion of upper and lower probabilities induced by a random set:

Definition 10. [? ] Let $(\Omega, \mathcal{A}, P)$ be a probability space, $\left(\Omega^{\prime}, \mathcal{A}\right)$ a measurable space and $\Gamma: \Omega \rightarrow \mathcal{P}\left(\Omega^{\prime}\right)$ a random set. Then its upper and lower probabilities are the functions $P^{*}, P_{*}: \mathcal{A} \rightarrow[0,1]$ given by $P^{*}(A)=P(\{\omega: \Gamma(\omega) \cap A \neq \emptyset\})$ and $P_{*}(A)=P(\{\omega: \emptyset \neq \Gamma(\omega) \subseteq A\})$ for every $A \in \mathcal{A}$.

The upper and lower probabilities of a random set are in particular coherent lower and upper probabilities as introduced in Section ??, and constitute upper and lower bounds of the probabilities induced by the measurable selections:

$$
P_{*}(A) \leq P_{X}(A) \leq P^{*}(A) \text { for every } X \in S(\Gamma) ;
$$

therefore, their associated cumulative distributions provide lower and upper bounds of the lower and upper distribution functions associated to $S(\Gamma)$. The inequalities in Eq. (??) may be strict [? , Example 1]; however, under fairly general conditions

$$
P^{*}(A)=\max \mathcal{P}(\Gamma)(A) \text { and } P_{*}(A)=\min \mathcal{P}(\Gamma)(A) \text { for every } A \in \mathcal{A}^{\prime},
$$

where $\mathcal{P}(\Gamma)(A):=\left\{P_{X}(A): X \in S(\Gamma)\right\}$. In particular, if $\Gamma$ takes values on the measurable space $\left([0,1], \beta_{[0,1]}\right)$, where $\beta_{[0,1]}$ denotes the Borel $\sigma$-field, Eq. (??) holds under any of the following conditions [? ]:

- If the class $\{\Gamma(\omega): \omega \in \Omega\}$ is countable;

- if $\Gamma(\omega)$ is closed for every $\omega \in \Omega$;

- if $\Gamma(\omega)$ is open for every $\omega \in \Omega$.

Theorem 13. [? , Theorem 14] Let $(\Omega, \mathcal{A}, P)$ be a probability space. Consider the measurable space $\left([0,1], \beta_{[0,1]}\right)$ and let $\Gamma: \Omega \rightarrow \mathcal{P}([0,1])$ be a random set. If 
$P^{*}(A)=\max \mathcal{P}(\Gamma)(A)$ for all $A \in \mathcal{A}^{\prime}$, then for any bounded random variable $f:[0,1] \rightarrow \mathbb{R}:$

$$
(C) \int f d P^{*}=\sup _{X \in S(\Gamma)} \int f d P_{\mathrm{X}}, \quad(C) \int f d P_{*}=\inf _{X \in S(\Gamma)} \int f d P_{\mathrm{X}},
$$

and consequently:

$$
(C) \int f d P^{*}=\sup (A) \int(f \circ \Gamma) d P, \quad(C) \int f d P_{*}=\inf (A) \int(f \circ \Gamma) d P,
$$

where $(C) \int f d P^{*}$ denotes the Choquet integral of $f$ with respect to $P^{*}$, and (A) $\int(f \circ \Gamma) d P$ denotes the Aumann integral of $f \circ \Gamma$ with respect to $P$.

These results allow us to characterize the imprecise stochastic dominance between random sets by means of the comparison of Choquet or Aumann integrals. Recall that we have denoted by $\mathcal{U}^{*}$ the set of non-decreasing and bounded random variables $u:[0,1] \rightarrow \mathbb{R}$.

Proposition 14. Let $(\Omega, \mathcal{A}, P)$ be a probability space. Consider the measurable space $\left([0,1], \beta_{[0,1]}\right)$ and let $\Gamma_{1}, \Gamma_{2}: \Omega \rightarrow \mathcal{P}([0,1])$ be two random sets. If $P_{1}^{*}(A)=$ $\max \mathcal{P}\left(\Gamma_{1}\right)(A)$ and $P_{2}^{*}(A)=\max \mathcal{P}\left(\Gamma_{2}\right)(A)$ for all $A \in \beta_{[0,1]}$, the following equivalences hold:
1. $\Gamma_{1} \succeq_{\mathrm{FSD}_{1}} \Gamma_{2} \Leftrightarrow(C) \int u d P_{1 *} \geq(C) \int u d P_{2}^{*}$ for every $u \in \mathcal{U}^{*}$.
2. $\Gamma_{1} \succeq_{\mathrm{FSD}_{2}} \Gamma_{2} \Rightarrow(C) \int u d P_{1}^{*} \geq(C) \int u d P_{2}^{*}$ for every $u \in \mathcal{U}^{*}$.
3. $\Gamma_{1} \succeq_{\mathrm{FSD}_{3}} \Gamma_{2} \Rightarrow(C) \int u d P_{1}^{*} \geq(C) \int u d P_{2}^{*}$ for every $u \in \mathcal{U}^{*}$.
4. $\Gamma_{1} \succeq_{\mathrm{FSD}_{4}} \Gamma_{2} \Rightarrow(C) \int u d P_{1}^{*} \geq(C) \int u d P_{2 *}$ for every $u \in \mathcal{U}^{*}$.
5. $\Gamma_{1} \succeq_{\mathrm{FSD}_{5}} \Gamma_{2} \Rightarrow(C) \int u d P_{1 *} \geq(C) \int u d P_{2 *}$ for every $u \in \mathcal{U}^{*}$.
6. $\Gamma_{1} \succeq_{\mathrm{FSD}_{6}} \Gamma_{2} \Rightarrow(C) \int u d P_{1 *} \geq(C) \int u d P_{2 *}$ for every $u \in \mathcal{U}^{*}$.

Proof. Consider $u \in \mathcal{U}^{*}$. We deduce from Theorem ?? that, under the hypotheses of the proposition,

$$
\begin{aligned}
& (C) \int u d P_{i}^{*}=\sup _{X \in S\left(\Gamma_{i}\right)} \int u d P_{\mathrm{X}}=\bar{E}_{S\left(\Gamma_{i}\right)}(u) \text { and } \\
& (C) \int u d P_{i *}=\inf _{X \in S\left(\Gamma_{i}\right)} \int u d P_{\mathrm{X}}=\underline{E}_{S\left(\Gamma_{i}\right)}(u)
\end{aligned}
$$

for $i=1,2$. The result follows then applying Theorem ??.

Let us discuss next the comparison of random sets by means of statistical preference. When the utility space $\Omega^{\prime}$ is finite, we obtain a result akin to Proposition ??: 
Proposition 15. Let $(\Omega, \mathcal{A}, P)$ be a probability space, $\left(\Omega^{\prime}, \mathcal{P}\left(\Omega^{\prime}\right)\right)$ a measurable space, with $\Omega^{\prime}$ finite, and $\Gamma_{1}, \Gamma_{2}$ be two random sets. The following equivalences hold:

(a) $\Gamma_{1} \succeq_{\mathrm{SP}_{1}} \Gamma_{2} \Leftrightarrow \min \Gamma_{1} \succeq_{\mathrm{SP}} \max \Gamma_{2}$.

(b) $\Gamma_{1} \succeq_{\mathrm{SP}_{2}} \Gamma_{2} \Leftrightarrow \Gamma_{1} \succeq_{\mathrm{SP}_{3}} \Gamma_{2} \Leftrightarrow \max \Gamma_{1} \succeq_{\mathrm{SP}} \max \Gamma_{2}$.

(c) $\Gamma_{1} \succeq_{\mathrm{SP}_{4}} \Gamma_{2} \Leftrightarrow \max \Gamma_{1} \succeq_{\mathrm{SP}} \min \Gamma_{2}$.

(d) $\Gamma_{1} \succeq_{\mathrm{SP}_{5}} \Gamma_{2} \Leftrightarrow \Gamma_{1} \succeq_{\mathrm{SP}_{6}} \Gamma_{2} \Leftrightarrow \min \Gamma_{1} \succeq_{\mathrm{SP}} \min \Gamma_{2}$.

Proof. The result follows from Proposition ??, taking into account that statistical preference satisfies the monotonicity condition of Eq. (??) and that if $\Gamma$ is a random set taking values on a finite space, then the mappings $\min \Gamma, \max \Gamma$ belong to $S(\Gamma)$.

In particular, we deduce that we can focus on the minimum and maximum measurable selections in order to characterize these extensions of statistical preference:

Corollary 16. Let $(\Omega, \mathcal{A}, P)$ be a probability space, $\Omega^{\prime}$ a finite space and let $\Gamma_{1}, \Gamma_{2}: \Omega \rightarrow \mathcal{P}\left(\Omega^{\prime}\right)$ be two random sets. Then for every $i=1, \ldots, 6$ :

$$
\Gamma_{1} \succeq_{\mathrm{SP}_{\mathrm{i}}} \Gamma_{2} \Leftrightarrow\left\{\min \Gamma_{1}, \max \Gamma_{1}\right\} \succeq_{\mathrm{SP}_{\mathrm{i}}}\left\{\min \Gamma_{2}, \max \Gamma_{2}\right\} .
$$

These two results are interesting because random sets taking values on finite spaces are quite common in practice; they have been studied in detail in [? ? ], and one of their most interesting properties is that they constitute equivalent models to belief and plausibility functions [? ].

Note that the equivalence in Eq. (??) does not hold for the relation $\succeq_{\mathrm{SP}_{\text {mean }}}$ defined in Section ??:

Example 4. Consider the probability space $(\Omega, \mathcal{A}, P)$ where $\Omega=\left\{\omega_{1}, \omega_{2}\right\}, \mathcal{A}=$ $\mathcal{P}(\Omega)$ and $P$ is the probability distribution given by $P\left(\left\{\omega_{1}\right\}\right)=P\left(\left\{\omega_{2}\right\}\right)=0.5$, and let $\Gamma_{1}$ be the random set given by $\Gamma_{1}\left(\omega_{1}\right)=\{0,1\}, \Gamma_{1}\left(\omega_{2}\right)=\{0,2,3,4\}$, and let $\Gamma_{2}$ be single-valued random set given by $\Gamma_{2}\left(\omega_{1}\right)=\{1\}=\Gamma_{2}\left(\omega_{2}\right)$. Then $\min \Gamma_{1}$ is the constant random variable on 0 , while $\max \Gamma_{1}$ is given by $\max \Gamma_{1}\left(\omega_{1}\right)=$ $1, \max \Gamma_{1}\left(\omega_{2}\right)=4$. Hence, if we compare $\left\{\min \Gamma_{1}, \max \Gamma_{1}\right\}$ with $\Gamma_{2}$ by means of $\succeq_{\mathrm{SP}_{\text {mean }}}$ we obtain

$$
\frac{Q\left(\min \Gamma_{1}, \Gamma_{2}\right)+Q\left(\max \Gamma_{1}, \Gamma_{2}\right)}{2}=\frac{0+0.75}{2}=0.375
$$

and thus $\Gamma_{2} \succ_{\mathrm{SP}_{\text {mean }}}\left\{\min \Gamma_{1}, \max \Gamma_{1}\right\}$. On the other hand, the set of selections of $\Gamma_{1}$ is given by (where a selection $X$ is identified with the vector $\left.\left(X\left(\omega_{1}\right), X\left(\omega_{2}\right)\right)\right): S\left(\Gamma_{1}\right)=\{(0,0),(0,2),(0,3),(0,4),(1,0),(1,2),(1,3),(1,4)\}$, from which we deduce that $\Gamma_{1} \succ_{\mathrm{SP}_{\text {mean }}} \Gamma_{2}$. 


\section{Comparison of alternatives with imprecise probabilities}

We next consider the case where we want to choose between two alternatives $X, Y: \Omega \rightarrow \Omega^{\prime}$, and there is some uncertainty about the probability distribution $P$ of the different states of nature $\omega \in \Omega$, that we model by means of a set $\mathcal{P}$ of probability distributions on $\Omega$. Then we may associate to $X$ a set $\mathcal{X}$ of random variables, which correspond to the transformations of $X$ under any of the probability distributions in $\mathcal{P}$; and similarly for $Y$. We end up thus with two sets $\mathcal{X}, \mathcal{Y}$ of random variables, and we should establish methods to determine which of these two sets is preferable ${ }^{1}$.

In this situation, we may compare the sets $\mathcal{X}, \mathcal{Y}$ by means of the generalizations of statistical preference or stochastic dominance we have discussed in Section ??; however, we argue that other notions may make more sense in this context. This is because conditions $\succeq_{1}, \ldots, \succeq_{6}$ are based on considering a particular pair $\left(X_{1}, Y_{1}\right)$ in $\mathcal{X} \times \mathcal{Y}$ and on comparing $X_{1}$ with $Y_{1}$ by means of the binary relation $\succeq$. However, any $X_{1}$ in $\mathcal{X}$ corresponds to a particular choice of a probability measure $P \in \mathcal{P}$, and similarly for any $Y_{1} \in \mathcal{Y}$; and if we use an epistemic interpretation of our uncertainty under which only one $P \in \mathcal{P}$ is the 'true' model, it makes no sense to compare $X_{1}$ and $Y_{1}$ based on a different distribution. This is particularly clear in case we want to apply statistical preference, which is based on comparing $P(X>Y)$ with $P(Y>X)$, where $P$ is the initial probability measure.

To make this clearer, in this section we may denote our sets of alternatives $\mathcal{X}:=\{(X, P): P \in \mathcal{P}\}$ and $\mathcal{Y}:=\{(Y, P): P \in \mathcal{P}\}$, meaning that our utilities are precise (and are determined by the variables $X$ and $Y$, respectively), while our beliefs are imprecise and are modeled by the set $\mathcal{P}$. To avoid confusions, we will now write $X \succeq^{P} Y$ to express that $X$ is preferred to $Y$ when we consider the probability measure $P$ in the initial probability space. Then we can establish the following definitions ${ }^{2}$ :

Definition 11. Let $\succeq$ be a binary relation on random variables. We say that:

- $\mathcal{X}$ is strongly $\mathcal{P}$ preferred to $\mathcal{Y}$, and denote it $\mathcal{X} \succeq_{\mathrm{s}}^{\mathcal{P}} \mathcal{Y}$, when $X \succeq^{P} Y$ for every $P \in \mathcal{P}$;

\footnotetext{
${ }^{1}$ One particular case where this situation may arise is in the context of missing data [? ]. We may divide the variables determining the states of nature in two groups: one for which we have precise information, that we model by means of a probability measure $P^{\prime}$ over the different states, and another one about which are completely ignorant, knowing only the values they take, and nothing more. Then we may get to the classical scenario by fixing the value of the variables in this second group: for each of these values the alternatives may be seen as random variables, using the probability measure $P^{\prime}$ to determine the probabilities of the different rewards. Hence, by doing this we would transform the two alternatives $X$ and $Y$ into two sets of alternatives $\mathcal{X}, \mathcal{Y}$, considering all the possible values of the variables in the second group.

${ }^{2}$ A similar idea can be found in [? ] in the context of robust ordinal regression; in that case, the set of additive value functions compatible with the available information is obtained and two binary relations between pairs of alternatives are defined, depending on whether an alternative is preferred to another one with respect to all or some of the value functions.
} 
- $\mathcal{X}$ is weakly $\mathcal{P}$ preferred, and denote it $\mathcal{X} \succeq_{\mathrm{w}}^{\mathcal{P}} \mathcal{Y}$, to $\mathcal{Y}$ when $X \succeq^{P} Y$ for some $P \in \mathcal{P}$.

Obviously, the strong preference implies the weak one. To see that they are not equivalent, consider the following simple example:

Example 5. Let $\succeq$ be the binary relation associated to statistical preference and consider the variables $X, Y$ in Example ??. If we consider the uniform distribution $P_{1}$ in all the die outcomes, we obtain $Q(X, Y)=\frac{20}{36}$, so that $X \succeq_{\mathrm{SP}}^{P_{1}}$ $Y$; if we take instead the uniform distribution $P_{2}$ on the first three outcomes, then $Q(X, Y)=\frac{2}{9}$, and as a consequence $Y \succeq_{\mathrm{SP}}^{P_{2}} X$. Hence, $X$ is weakly $\left\{P_{1}, P_{2}\right\}$ statistically preferred to $Y$, but not strongly so.

With respect to the notions established in Section ??, it is not difficult to establish the following result. Its proof is immediate, and therefore omitted.

Proposition 17. Let $\mathcal{X}, \mathcal{Y}$ be the sets of alternatives considered above, and let $\succeq$ be a binary relation. Then $\mathcal{X} \succeq_{1} \mathcal{Y} \Rightarrow \mathcal{X} \succeq_{\mathrm{s}}^{\mathcal{P}} \mathcal{Y} \Rightarrow \mathcal{X} \succeq_{\mathrm{w}}^{\mathcal{P}} \mathcal{Y} \Rightarrow \mathcal{X} \succeq_{4} \mathcal{Y}$.

Let us show that the converse implications do not hold:

Example 6. Consider $\Omega=\left\{\omega_{1}, \omega_{2}, \omega_{3}\right\}, \mathcal{P}:=\left\{P: P\left(\omega_{1}\right)>P\left(\omega_{2}\right), P\left(\omega_{2}\right) \in\right.$ $[0,0.2]\}$ and the alternatives $X, Y$ given by

\begin{tabular}{l|lll} 
& $\omega_{1}$ & $\omega_{2}$ & $\omega_{3}$ \\
\hline$X$ & 1 & 0 & 1 \\
$Y$ & 0 & 1 & 1
\end{tabular}

Then if we consider the sets of alternatives $\mathcal{X}=\{(X, P): P \in \mathcal{P}\}$ and $\mathcal{Y}=$ $\{(Y, P): P \in \mathcal{P}\}$ and we compare them by means of stochastic dominance, it is clear that $\mathcal{X} \succ_{\mathrm{s}}^{\mathcal{P}} \mathcal{Y}$; however, it does not hold that $\mathcal{X} \succeq_{\mathrm{FSD}_{1}} \mathcal{Y}$ : if we consider $P_{1}:=(0.3,0.2,0.5)$ and $P_{2}:=(0.1,0,0.9)$, it holds that $\left(Y, P_{2}\right) \succ_{\mathrm{FSD}}\left(X, P_{1}\right)$.

Moreover, in this example we also have that $\mathcal{X}$ is strictly weakly $\mathcal{P}$-preferred to $\mathcal{Y}$ while $\mathcal{X} \equiv_{\mathrm{FSD}_{4}} \mathcal{Y}$.

Remark 4. If the binary relation $\succeq$ we start with is complete, so is the weak $\mathcal{P}$-preference. In that case, we obtain that $\mathcal{X} \succ_{\mathrm{w}}^{\mathcal{P}} \mathcal{Y}$ implies that $\mathcal{X} \succ_{\mathrm{s}}^{\mathcal{P}} \mathcal{Y}$, because if $\mathcal{X} \succ_{\mathrm{w}}^{\mathcal{P}} \mathcal{Y}$ we must have that $(X, P) \succ(Y, P)$ for every $P \in \mathcal{P}$.

Moreover, when $\mathcal{X} \equiv_{\mathrm{w}}^{\mathcal{P}} \mathcal{Y}$, we may have strict preference, indifference or incomparability with respect to strong $\mathcal{P}$-preference.

In what follows, we study in some detail the notions of weak and strong preference for particular choices of the binary relation $\succeq$. If $\succeq$ corresponds to expected utility, strong preference of $\mathcal{X}$ over $\mathcal{Y}$ means that $X$ is preferred to $Y$ with respect to all the probability measures $P$ in $\mathcal{P}$, and is thus related to the idea of maximality [? ]; on the other hand, weak preference means that $X$ is preferred to $Y$ (i.e., it is the optimal alternative) with respect to some of the elements of $\mathcal{P}$; this idea is close to the criterion of $E$-admissibility [? ]. See also Remark ?? and [? , Section 3.2].

When $\succeq$ is the binary relation associated to stochastic dominance, we obtain the following: 
Proposition 18. Consider a set $\mathcal{P}$ of probability measures on $\Omega$, and let $X, Y$ be two real-valued random variables on $\Omega$. Let us define $\mathcal{F}_{\mathrm{X}}:=\left\{F_{\mathrm{X}}^{P}: P \in \mathcal{P}\right\}$ and $\mathcal{F}_{\mathrm{Y}}:=\left\{F_{\mathrm{Y}}^{P}: P \in \mathcal{P}\right\}$, and $\left(\underline{F}_{\mathrm{X}}, \bar{F}_{\mathrm{X}}\right),\left(\underline{F}_{\mathrm{Y}}, \bar{F}_{\mathrm{Y}}\right)$ their associated p-boxes.

1. $\bar{F}_{\mathrm{X}} \leq \underline{F}_{\mathrm{Y}} \Rightarrow \mathcal{X}$ is strongly $\mathcal{P}$-preferred to $\mathcal{Y}$ with respect to stochastic dominance.

2. $\mathcal{X}$ is weakly $\mathcal{P}$-preferred to $\mathcal{Y}$ with respect to stochastic dominance $\Rightarrow \underline{F}_{\mathrm{X}} \leq$ $\bar{F}_{\mathrm{Y}}$.

Proof. The result follows from Propositions ?? and ??.

Finally, when $\succeq$ corresponds to statistical preference, we can apply Remark ??, because $\succeq$ is a complete relation. In addition, we can establish the following result:

Proposition 19. Consider a set $\mathcal{P}$ of probability measures, and let $\underline{P}, \bar{P}$ denote its lower and upper envelopes, given by Eq. (??). Let $X, Y$ be two real-valued random variables on $\Omega$, and let $u=I_{(0,+\infty)}-I_{(-\infty, 0)}$.

1. $X$ is strongly $\mathcal{P}$ statistically preferred to $Y \Leftrightarrow \underline{P}(u(X-Y)) \geq 0$.

2. $X$ is weakly $\mathcal{P}$ statistically preferred to $Y \Rightarrow \bar{P}(u(X-Y)) \geq 0$. The converse holds if $\mathcal{P}=\mathcal{M}(\bar{P})$.

Proof. The result follows simply by considering that if $X, Y$ are random variables on a probability space $(\Omega, \mathcal{A}, P)$, then, by Eq. (??) $X \succeq_{\mathrm{SP}}^{P} Y$ if and only if $P(u(X-Y)) \geq 0$, where we also use $P$ to denote the expectation operator associated to the probability measure $P$.

To see that the converse of the second statement holds when $\mathcal{P}=\mathcal{M}(\underline{P})$, note that the upper envelope $\bar{P}$ of $\mathcal{P}$ is a coherent lower prevision. From [? , Section 3.3.3], given the bounded random variable $u(X-Y)$ there is some $P \in \mathcal{M}(\underline{P})$ such that $P(u(X-Y))=\bar{P}(u(X-Y))$.

\section{Imprecise preferences applied to decision making}

In this section, we shall illustrate the previous results by means of an application to decision making. We shall consider two different scenarios: on the one hand, we shall compare two alternatives in a context of imprecise information about their utilities or probabilities, by means of the results in Sections ?? and ??; on the other hand, we shall consider the comparison of two sets of alternatives, by means of the techniques established in Section ??. Our running example throughout this section is based on [? , Section 4].

\subsection{A decision problem with uncertain beliefs}

Let us consider a decision problem where we must choose between $n$ alternatives $a_{1}, \ldots, a_{n}$, whose rewards depend on the values of the states of nature, $\theta_{1}, \ldots, \theta_{m}$, which hold with certain probabilities $P\left(\theta_{1}\right), \ldots, P\left(\theta_{m}\right)$.

Let us start by assuming that there is uncertainty about these probabilities, that we model by means of a set of probability measures $\mathcal{P}$. Then, we shall compare any two alternatives by means of the concepts of weak and strong $\mathcal{P}$-preference we have considered in Section ??. 
Example 7. A company must choose where to invest its money. The alternatives are: $a_{1}-$ a computer company; $a_{2}-a$ car company; $a_{3}-a$ fast food company. The rewards associated to the investment depend on an attribute $c_{1}$ : "economic evolution", which may take the values $\theta_{1}$-"very good", $\theta_{2}$-"good", $\theta_{3}$-"normal" or $\theta_{4}$ - "bad". The probabilities of each of these states are expressed by means of an interval. The rewards associated to any combination (alternative, state) are expressed in a linguistic scale, with values $S=\left\{s_{0}, s_{1}, s_{2}, s_{3}, s_{4}, s_{5}, s_{6}\right\}$ (very poor, poor, slightly poor, normal, slightly good, good, very good). The available information is summarized in the following table:

\begin{tabular}{ccccc} 
& $\theta_{1}$ & $\theta_{2}$ & $\theta_{3}$ & $\theta_{4}$ \\
\hline & {$[0.1,0.4]$} & {$[0.2,0.7]$} & {$[0.3,0.4]$} & {$[0.1,0.5]$} \\
$a_{1}$ & $s_{4}$ & $s_{3}$ & $s_{3}$ & $s_{2}$ \\
$a_{2}$ & $s_{5}$ & $s_{4}$ & $s_{4}$ & $s_{2}$ \\
$a_{3}$ & $s_{2}$ & $s_{3}$ & $s_{5}$ & $s_{4}$
\end{tabular}

Hence, the set $\mathcal{P}$ of probability measures for our beliefs is given by

$$
\begin{aligned}
\mathcal{P}=\{ & \left(p_{1}, p_{2}, p_{3}, p_{4}\right): p_{1}+p_{2}+p_{3}+p_{4}=1, \\
& \left.p_{1} \in[0.1,0.4], p_{2} \in[0.2,0.7], p_{3} \in[0.3,0.4], p_{4} \in[0.1,0.5]\right\} .
\end{aligned}
$$

Since the rewards are expressed in a qualitative scale, we are going to compare the different alternatives by means of statistical preference. We obtain that:

$$
\begin{aligned}
& Q\left(a_{1}, a_{2}\right)=\frac{1}{2} p_{4} \in[0.05,0.25] . \quad Q\left(a_{1}, a_{3}\right)=p_{1}+\frac{1}{2} p_{2} \in[0.2,0.5] . \\
& Q\left(a_{2}, a_{3}\right)=p_{1}+p_{2} \in[0.3,0.6] \text {. }
\end{aligned}
$$

We deduce that, using statistical preference as our basic binary relation:

- $a_{2} \succ_{\mathrm{s}}^{\mathcal{P}} a_{1}$ and $a_{2} \succ_{\mathrm{w}}^{\mathcal{P}} a_{1}$.

- $a_{3} \succ_{\mathrm{s}}^{\mathcal{P}} a_{1}$ and $a_{3} \equiv_{\mathrm{w}}^{\mathcal{P}} a_{1}$.

- $a_{2} \equiv_{\mathrm{w}}^{\mathcal{P}} a_{3}$ and they are incomparable with respect to strong $\mathcal{P}$-preference.

Consequently, with respect to the strong preference, both the car company and the fast food companies are preferred to the computer company, and they are incomparable to each other. With respect to the weak preference, the car company is also preferred to the computer company, while the fast food company is indifferent to the car and the computer companies.

\subsection{A decision problem with uncertain rewards}

Let us assume next that we have precise information about the probabilities of the different states of nature but that we have imprecise information about the utilities associated to the different rewards. Let us model this case by means of a random set, as we discussed in Section ??. 
Example ?? (Cont). Assume that the probability of the different states of nature is given by:

$$
P\left(\theta_{1}\right)=0.2 \quad P\left(\theta_{2}\right)=0.25 \quad P\left(\theta_{3}\right)=0.3 \quad P\left(\theta_{4}\right)=0.25,
$$

but that we cannot determine precisely the consequences associated to each combination (alternative, state). We model the available information by means of a set of possible consequences, that we summarize in the following table:

\begin{tabular}{ccccc} 
& $\theta_{1}$ & $\theta_{2}$ & $\theta_{3}$ & $\theta_{4}$ \\
\hline & 0.2 & 0.25 & 0.3 & 0.25 \\
$a_{1}$ & $\left\{s_{4}, s_{5}\right\}$ & $\left\{s_{3}\right\}$ & $\left\{s_{2}, s_{3}\right\}$ & $\left\{s_{2}\right\}$ \\
$a_{2}$ & $\left\{s_{5}\right\}$ & $\left\{s_{3}, s_{4}\right\}$ & $\left\{s_{3}, s_{5}\right\}$ & $\left\{s_{2}, s_{4}\right\}$ \\
$a_{3}$ & $\left\{s_{2}\right\}$ & $\left\{s_{3}\right\}$ & $\left\{s_{3}, s_{5}\right\}$ & $\left\{s_{3}, s_{4}\right\}$
\end{tabular}

Since again we have qualitative rewards, we shall use statistical preference to compare the different alternatives. Taking into account that the utility space is finite, we deduce from Proposition ?? that the comparison of the random sets associated to each of the alternatives reduces to the comparison of the greatest and smallest measurable selections. Moreover, since the utility space is finite, $\succeq_{\mathrm{SP}_{2}} \Leftrightarrow \succeq_{\mathrm{SP}_{3}}$ and $\succeq_{\mathrm{SP}_{5}} \Leftrightarrow \succeq_{\mathrm{SP}_{6}}$.

Let us compare alternatives $a_{1}, a_{2}$ :

$$
\begin{aligned}
& Q\left(\min a_{1}, \max a_{2}\right)=0 . \quad Q\left(\min a_{1}, \min a_{2}\right)=0.25 . \\
& Q\left(\max a_{1}, \max a_{2}\right)=0.1 . \quad Q\left(\max a_{1}, \min a_{2}\right)=0.5 \text {. }
\end{aligned}
$$

Using Proposition ??, we conclude that $a_{2} \succ_{\mathrm{SP}_{\mathrm{i}}} a_{1}$ for $i=1,2,3,5,6$ and $a_{1} \equiv_{\mathrm{SP}_{4}} a_{2}$.

With respect to alternatives $a_{1}$ and $a_{3}$, we obtain that:

$$
\begin{array}{ll}
Q\left(\min a_{1}, \max a_{3}\right)=0.325 . & Q\left(\min a_{1}, \min a_{3}\right)=0.325 . \\
Q\left(\max a_{1}, \max a_{3}\right)=0.325 . & Q\left(\max a_{1}, \min a_{3}\right)=0.475 .
\end{array}
$$

Using Proposition ??, we conclude that $a_{3} \succ_{\mathrm{SP}_{\mathrm{i}}} a_{1}$ for $i=4$ and as a consequence also for $i=1,2,3,5,6$.

Finally, if we compare alternatives $a_{2}$ and $a_{3}$, we obtain that:

\begin{tabular}{|c|c|c|c|c|c|c|}
\hline & $S P_{1}$ & $S P_{2}$ & $S P_{3}$ & $S P_{4}$ & $S P_{5}$ & $S P_{6}$ \\
\hline$\left(a_{1}, a_{2}\right)$ & $\prec_{\mathrm{SF}}$ & $\prec_{\mathrm{SP}_{2}}$ & $\prec \mathrm{SP}_{3}$ & $\equiv \mathrm{SP}_{4}$ & $\prec_{\mathrm{SP}_{5}}$ & $\prec_{\mathrm{SP}_{6}}$ \\
\hline$\left(a_{1}, a_{3}\right)$ & $\prec_{\mathrm{SP}_{1}}$ & $\prec_{\mathrm{SP}_{2}}$ & $\prec \mathrm{SP}_{3}$ & $\prec_{\mathrm{SP}_{4}}$ & $\prec_{\mathrm{S}}$ & $\prec_{\mathrm{SP}_{6}}$ \\
\hline$\left(a_{2}, a_{3}\right)$ & $\nsim_{\mathrm{SP}_{1}}$ & $\succ_{\mathrm{SP}_{2}}$ & $\succ \mathrm{SP}_{3}$ & $\equiv_{\mathrm{SP}_{4}}$ & $\prec_{\mathrm{SP}_{5}}$ & $\prec_{\mathrm{SP}}$ \\
\hline
\end{tabular}

$$
\begin{array}{ll}
Q\left(\min a_{2}, \max a_{3}\right)=0.325 . & Q\left(\min a_{2}, \min a_{3}\right)=0.475 . \\
Q\left(\max a_{2}, \max a_{3}\right)=0.725 . & Q\left(\max a_{2}, \min a_{3}\right)=1 .
\end{array}
$$

Using Proposition ??, we conclude that $a_{2} \succ_{\mathrm{SP}_{\mathrm{i}}} a_{3}$ for $i=2,3, a_{3} \succ_{\mathrm{SP}_{\mathrm{i}}} a_{2}$ for $i=5,6, a_{2} \equiv_{\mathrm{SP}_{4}} a_{3}$ and they are incomparable with respect to $\succeq_{\mathrm{SP}_{1}}$.

The above relationships are summarized in the following table:

Hence, both the car and the fast food companies are preferred to the computer one. On the other hand, the preference between the car and fast food companies would depend on our attitude towards risk, which would determine if we focus on the best or the worst-case scenarios. 


\subsection{A decision problem between sets of alternatives}

Assume now that we have precise beliefs and utilities but the choice must be made between sets of alternatives instead of pairs. In that case, we shall apply the conditions and results from Section ??.

Example ?? (Cont). Assume now that we may invest our money in another company $a_{4}$ in the telecommunications area, and that the choice must be made between two portfolios: one -that we shall denote $\mathcal{X}$-made by alternatives $a_{1}, a_{2}$, and another-denoted by $\mathcal{Y}$-made by $a_{3}, a_{4}$. Assume that the rewards associated to each alternative are given by the following table:

\begin{tabular}{lllll} 
& $\theta_{1}$ & $\theta_{2}$ & $\theta_{3}$ & $\theta_{4}$ \\
\hline & 0.2 & 0.25 & 0.3 & 0.25 \\
$a_{1}$ & 75 & 60 & 55 & 50 \\
$a_{2}$ & 80 & 65 & 55 & 40 \\
$a_{3}$ & 60 & 55 & 50 & 55 \\
$a_{4}$ & 80 & 55 & 40 & 65
\end{tabular}

where the utilities are now expressed in a $[0,100]$ scale.

If we compare these alternatives by means of stochastic dominance, we obtain that $a_{1} \succ_{\mathrm{FSD}} a_{3}, a_{2} \succ_{\mathrm{FSD}} a_{4}$ and any other pair $\left(a_{i}, a_{j}\right)$ with $i \in\{1,2\}, j \in$ $\{3,4\}$ are incomparable with respect to stochastic dominance. Hence, $\mathcal{X} \succ_{\mathrm{FSD}_{\mathrm{i}}} \mathcal{Y}$ for $i=3,4,6$ and they are incomparable with respect to $\succeq_{\mathrm{FSD}_{\mathrm{i}}}$ for $i=1,2,5$.

Note that this example is an instance where $\succeq_{\mathrm{FSD}_{2}}$ is not equivalent to $\succeq_{\mathrm{FSD}_{3}}$ and $\succeq_{\mathrm{FSD}_{5}}$ is not equivalent to $\succeq_{\mathrm{FSD}_{6}}$, because there is neither a maximum nor a minimum in the sets of distribution functions associated to $\mathcal{X}, \mathcal{Y}$.

On the other hand, if we compare the sets $\mathcal{X}, \mathcal{Y}$ by means of statistical preference, we obtain the following profile of preferences:

$$
\mathbb{Q}_{\mathcal{X}, \mathcal{Y}}:=\left(\begin{array}{ll}
0.75 & 0.55 \\
0.75 & 0.65
\end{array}\right) .
$$

From this we deduce that $\mathcal{X} \succ \mathrm{SP}_{1} \mathcal{Y}$, and as a consequence $\mathcal{X} \succ \mathrm{SP}_{\mathrm{i}} \mathcal{Y}$ for $i=2, \ldots, 6$ and also $\mathcal{X} \succ_{\mathrm{SP}_{\text {mean }}} \mathcal{Y}$. Hence, from the point of view of statistical preference the first portfolio should be preferred to the second.

\section{Conclusions}

In this paper, we have presented a number of choice models for decision making under uncertainty when there is imprecision about the probabilities of the states of nature and the utilities of the different alternatives. By considering all the probability measures and utility functions compatible with the available information, any alternative becomes a set of alternatives, and therefore the choice functions between pairs of alternatives must be extended in order to be able to choose between sets of alternatives.

We have proposed a number of choice models that accommodate different attitudes towards risk, by focusing on particular alternatives within each of the 
sets. These extensions can be applied to any choice function between pairs of alternatives. We have focused in particular on two such choice functions: stochastic dominance and statistical preference. The first one has been applied extensively on economics, while the second one is interesting when the utilities are expressed in a qualitative scale. Both of them serve as an alternative to the expected utility model, for which we have commented briefly on some of its possible generalizations to an imprecise context; they may also be regarded as more robust: in the case of stochastic dominance, it is invariant under increasing transformations of the utility function; on the other hand, statistical preference is related to the median, and is less affected by extreme rewards of the alternatives.

We think that with these relationships we are now able to effectively choose between two alternatives in any of the possible scenarios of imprecision: in either the probabilities of the states of nature or the utilities of the different alternatives; for qualitative or quantitative utility scales; and for different attitudes towards risk, more or less conservative.

From our results, there are a number of open problems which may be of interest: the comparison of more than two sets of alternatives, by means of transitive choice functions; the study of the stochastic dominance of the $n$-th order; the modeling of imprecise beliefs and utilities by means of random sets with an imprecise probability model on the initial space; and a deeper study of the connection with the work on robust ordinal regression.

\section{Acknowledgements}

Work partially supported by project MTM2010-17844, and by the Science and Education Ministry FPU grant AP2009-1034. We would also like to thank Didier Dubois and the editor for some helpful comments.

\section{References}

[1] R. J. Aumann. Utility theory without the completeness axiom. Econometrica, 30:445-462, 1962.

[2] D. Batur and F. Choobineh. Stochastic dominance based comparison for system selection. European Journal of Operational Research, 220:661-672, 2012 .

[3] J. C. Bezdek, B. Spillman and R. Spillman. A fuzzy relation space for group decision theory. Fuzzy Sets and Systems 1:255-268, 1978.

[4] P. J. Boland, M. Hollander, K. Joag-Kev and S. Kochar. Bivariate dependence properties of order statistics. Journal of Multivariate Analysis, $56: 75-89,1996$.

[5] T. Calvo, A. Kolesárová, M. Komorníková, and R. Mesiar. Aggregation operators. New Trends and Applications. Physica-Verlag, Heidelbert, 2002. 
[6] G. Choquet. Theory of capacities. Annales de l'Institut Fourier, 5:131-295, 1953-1954.

[7] M. Condorcet. An essay on the application of probability theory to plurality decision making: an election between three candidates (1785). Reprinted in F. Sommerlad, I. McLean (Eds.), pages 69-80. 1989.

[8] I. Couso and D. Dubois. An imprecise probability approach to joint extensions of stochastic and interval orderings. In Proceedings of IPMU 2012, pages 388-399. Springer-Verlag, 2012.

[9] I. Couso and L. Sánchez. The behavioural meaning of the median. In C. Borgelt, G. González-Rodríguez, W. Trutschnig, M.A. Lubiano, M.A. Gil, P. Grzegiorzewski, and O. Hryniewicz, editors, Combining Soft Computing and Statistical Methods in Data Analysis, volume 77 of Advances in Intelligent and Soft Computing, pages 115-122. Springer, 2010.

[10] B. De Schuymer, H. De Meyer, and B. De Baets. A fuzzy approach to stochastic dominance of random variables, pages 253-260. Lecture Notes in Artificial Intelligence 2715, 2003.

[11] B. De Schuymer, H. De Meyer, B. De Baets, and S. Jenei. On the cycletransitivity of the dice model. Theory and Decision, 54:261-285, 2003.

[12] A. P. Dempster. Upper and lower probabilities induced by a multivalued mapping. Annals of Mathematical Statistics, 38:325-339, 1967.

[13] M. Denuit, J. Dhaene, M. Goovaerts, and R. Kaas. Actuarial theory for dependent risks. John Wiley \& Sons, 2005.

[14] D. Dubois, H. Fargier, and P. Perny. Qualitative decision theory with preference relations and comparative uncertainty: An axiomatic approach. Artificial Intelligence, 148:219-260, 2003.

[15] D. Dubois and H. Prade. Gradualness, uncertainty and bipolarity: Making sense of fuzzy sets. Fuzzy Sets and Systems, 192:3-24, 2012.

[16] J. Dupacova and M. Kopa. Robustness of optimal portfolios under risk and stochastic dominance. European Journal of Operational Research, 2013. In press.

[17] S. Ferson, V. Kreinovich, L. Ginzburg, D. S. Myers, and K. Sentz. Constructing probability boxes and Dempster-Shafer structures. Technical Report SAND2002-4015, Sandia National Laboratories, January 2003.

[18] J. L. García-Lapresta and B. Llamazares. Majority decisions based on differences of votes. Journal of Mathematical Economics, 35:463-381, 2001.

[19] I. Gilboa and D. Schmeidler. Maxmin expected utility with a non-unique prior. Journal of Mathematical Economics, 18:141-153, 1989. 
[20] M. J. Goovaerts, R. Kaas, A. E. Van Heerwaarden, and T. Bauwelinckx. Effective Actuarial Methods. North Holland, 1990.

[21] S. Greco, V. Mousseau, R. Słowiński. Ordinal regression revisited: multiple criteria ranking using a set of additive value functions. European Journal of Operational Research, 191:415-425, 2008.

[22] S. Greco, R. Słowiński, J.R. Figueira and V. Mousseau. Robust ordinal regression. In Trends in Multiple Criteria Decision Analysis. International Series in Operational Research and Management of Science, vol. 142, pages 241-282, 2010.

[23] M. Kadziński, S. Greco and R. Słowiński. Extreme ranking analysis in robust ordinal regression. Omega, 40:488-501, 2012.

[24] M. Kadziński, S. Greco and R. Słowiński. Selection of a representative vaue function in robust multiple criteria ranking and choice. European Journal of Operational Research, 217:541-553, 2012.

[25] R. Kruse and K. D. Meyer. Statistics with vague data. D. Reidel Publishing Company, Dordrecht, 1987.

[26] I. Levi. The enterprise of knowledge. MIT Press, Cambridge, 1980.

[27] H. Levy. Stochastic dominance. Kluwer Academic Publishers, 1998.

[28] P. Lui, F. Jin, X. Zhang, Y. Su, and M.Wang. Research on the multiattribute decision-making under risk with interval probability based on prospects theory and the uncertain linguistic variables. Knowledge-Based Systems, 24:554-561, 2011.

[29] E. Miranda, I. Couso, and P. Gil. Upper probabilities and selectors of random sets. In P. Grzegorzewski, O. Hryniewicz, and M. A. Gil, editors, Soft methods in probability, statistics and data analysis, pages 126-133. Physica-Verlag, Heidelberg, 2002.

[30] E. Miranda, I. Couso, and P. Gil. Approximations of upper and lower probabilities by measurable selections. Information Sciences, 180:1407$1417,2010$.

[31] A. Müller and D. Stoyan. Comparison Methods for Stochastic Models and Risks. Wiley, 2002.

[32] I. Montes, D. Martinetti, S. Díaz, and S. Montes. A characterisation of statistical preference by means of the median. 2012. Submitted for publication.

[33] I. Montes, E. Miranda, and S. Montes. Stochastic dominance with imprecise information. Computational Statistics and Data Analysis, 2012. In press. 
[34] R. Nau. The shape of incomplete preferences. Annals of Statistics, $34(5): 2430-2448,2006$.

[35] H. T. Nguyen. An introduction to random sets. Chapman and Hall, 2006.

[36] D. Ríos Insua. On the foundations of decision analysis with partial information. Theory and Decision, 33:83-100, 1992.

[37] M. Roubens and Ph. Vincke. Preference Modelling. Springer-Verlag, Berlin, 1985 .

[38] J. K. Satia and R. E. Lave. Markovian decision processes with uncertain transition probabilities. Operations Research, 21:728-740, 1973.

[39] T. Seidenfeld, M. J. Schervish, and J B. Kadane. A representation of partially ordered preferences. The Annals of Statistics, 23:2168-2217, 1995.

[40] G. Shafer. A Mathematical Theory of Evidence. Princeton University Press, Princeton, NJ, 1976.

[41] M. C. M. Troffaes. Decision making under uncertainty using imprecise probabilities. International Journal of Approximate Reasoning, 45(1):1729, 2007.

[42] P. Walley. Statistical Reasoning with Imprecise Probabilities. Chapman and Hall, London, 1991.

[43] L. A. Zadeh. Fuzzy sets as a basis for a theory of possibility. Fuzzy Sets and Systems, 1:3-28, 1978.

[44] M. Zaffalon and E. Miranda. Conservative inference rule for uncertain reasoning under incompleteness. Journal of Artificial Intelligence Research, 34:757-821, 2009.

[45] M. Zaffalon, K. Wesnes, and O. Petrini. Reliable diagnoses of dementia by the naive credal classifier inferred from incomplete cognitive data. Artificial Intelligence in Medicine, 29:61-79, 2003. 